\title{
CYCLIC AMALGAMS, HNN EXTENSIONS, AND GARSIDE ONE-RELATOR GROUPS
}

\author{
MATTHIEU PICANTIN \\ This paper is dedicated to the memory of my friend and mentor, Patrick Dehornoy.
}

\begin{abstract}
Garside groups are a natural lattice-theoretic generalisation of the braid groups and spherical type Artin-Tits groups. Here we show that the class of Garside groups is closed under some free products with cyclic amalgamated subgroups. We deduce that every tree product of infinite cyclic groups is a Garside group. Moreover, we study those cyclic HNN extensions of Garside groups that are Garside groups as well. Using a theorem of Pietrowski, we conclude this paper by stating that a non-cyclic one-relator group is Garside if and only if its centre is nontrivial.
\end{abstract}

\section{INTRODUCTION}

Braid groups are well understood due to Brieskorn's presentation theorem and the subsequent structural study by Deligne and Brieskorn-Saito [6,20] : their main combinatorial results express that every braid group is the group of fractions of a monoid in which divisibility has good properties, and, in addition, there exists a distinguished element whose divisors encode the whole structure : in modern terminology, such a monoid is called Garside. The group of fractions of a Garside monoid is called a Garside group. Garside groups enjoy many remarkable group-theoretical, cohomological, and homotopy-theoretical properties [12,15].

The aim of this paper is to explore further the class of Garside groups. The latter happens to be closed under direct product and under some Zappa-Szép products. We just recall that a ZappaSzép product (or bi-crossed product, or knit product) of groups or monoids [50,51] is the natural extension of a semidirect product in which both groups (or monoids) act on one another, the semidirect product corresponding to the case when one action is trivial, and the direct product to the case when both actions are trivial. According to [15], the main general structural result about Garside structures known so far is that every Garside group is an iterated Zappa-Szép product of Garside groups with infinite cyclic centre [37].

Here we investigate the cyclic amalgamated free products of Garside monoids (Section 3). From this we deduce that every tree product of infinite cyclic groups is a Garside group. Then we characterise those cyclic HNN extensions of Garside groups that are again Garside groups (Section 44). As a nice consequence, using the solution of Pietrowski for the isomorphism problem for onerelator groups with nontrivial centre [44], we finally state that a non-cyclic one-relator group is Garside if and only if its centre is nontrivial (Section 5).

1991 Mathematics Subject Classification. 20E06 - 20F05 - 20F10 - 20F36.

Key words and phrases. amalgamated free product, HNN extension, tree product, knot group, braid monoid/group, one-relator group, Garside structure, automatic structure. 


\section{BACKGROUND FROM GARSIDE THEORY}

In this section, we list some basic properties of Garside monoids and groups. For all the results and the examples quoted here, we refer the reader to [15] (see also [12 14, 16, 36-41, 43]).

Assume that $M$ is a monoid. For $a, b$ in $M$, we say that $b$ is a left-divisor of $a-$ or that $a$ is a right-multiple of $b$-if $a=b d$ holds for some $d$ in $M$. An element $c$ is a lower common rightmultiple —or a right-lcm — of $a$ and $b$ if it is a right-multiple of both $a$ and $b$, and every common right-multiple of $a$ and $b$ is a right-multiple of $c$. Right-divisor, left-multiple, and left-lcm are defined symmetrically. For $a, b$ in $M$, we say that $b$ divides $a$-or that $b$ is a divisor of $a-$ if $a=c b d$ holds for some $c, d$ in $M$.

The monoid $M$ is said to be cancellative when, for any $a, b, c, d \in M, a b c=a d c$ implies $b=d$. And it is said to be conical if 1 is its only invertible element, that is, $a b=1$ implies $a=b=1$. Now, if $c, c^{\prime}$ are two right-lcms of $a$ and $b$, necessarily $c$ is a left-divisor of $c^{\prime}$, and $c^{\prime}$ is a leftdivisor of $c$. If we assume $M$ to be conical and cancellative, we have $c=c^{\prime}$. In this case, the unique right-lcm of $a$ and $b$ is denoted by $a \vee b$, and the unique element $c$ satisfying $a \vee b=a c$ is denoted by $a \backslash b$. We define the left-lcm $\widetilde{v}$ and the left operation / symmetrically. In particular, we have

$$
a \vee b=a(a \backslash b)=b(b \backslash a), \quad \text { and } \quad a \widetilde{v} b=(b / a) a=(a / b) b .
$$

Cancellativity and conicity imply that left- and right-divisibility are order relations.

Definition 2.1. A monoid $M$ is said to be Garside if $M$ is conical and cancellative, every pair of elements in $M$ admits a left-lcm and a right-lcm, and $M$ admits a Garside element, defined to be an element whose left- and right-divisors coincide, are finite in number, and generate $M$.

Example 2.2. By [6], all spherical type Artin-Tits monoids are Garside monoids. The classical braid monoids of the complex reflection groups $G_{7}, G_{11}, G_{12}, G_{13}, G_{15}, G_{19}$, and $G_{22}$ given in [7] (see [16, 36]), Garside's hypercube monoids [22,36], the dual braid monoids for spherical type Artin-Tits groups [3-5, 36, 39] and some so-called post-classical braid monoid [11] for complex reflection groups of type $(e, e, r)$ are also Garside monoids.

Definition 2.3. The enveloping group of a monoid $M$ is defined as the group admitting the same presentation as $M$; formally, it can be viewed as the monoid $\left\langle M \cup \bar{M}: R_{M} \cup F_{M}\right\rangle^{+}$, where $R_{M}$ is the family of all relations $b c=d$ with $b, c, d \in M$ and $F_{M}$ is the family of all relations $b \bar{b}=\bar{b} b=1$ with $b \in M$.

If $M$ is a Garside monoid, then $M$ satisfies Ore's conditions [9], and it embeds in a group of right-fractions, and, symmetrically, in a group of left-fractions. In this case, by construction, every right-fraction $a b^{-1}$ with $a, b$ in $M$ can be expressed as a left-fraction $c^{-1} d$ with $c, d$ in $M$, and conversely. Therefore, the two groups coincide, and there is no ambiguity in speaking of the group of fractions of a Garside monoid.

Definition 2.4. A group $G$ is said to be a Garside group if there exists a Garside monoid of which $G$ is the enveloping group, hence the group of fractions.

Example 2.5. Let us consider the monoid $M_{\chi}$ with presentation

$$
\left\langle a, b, c: a^{2}=b c, b^{3}=c a\right\rangle^{+} .
$$


The monoid $M_{\chi}$ is a typical example of a Garside monoid, and, in addition, $M_{\chi}$ has the distinguishing feature to not be anti-automorphic, contrary to those examples mentioned in Example 2.2. Its group of fractions happens to be the group $\left\langle a, b: a^{3}=b^{4}\right\rangle$ of the $(3,4)$-torus knot. We refer to [36, 40] for more Garside structures for torus link groups.

Remark 2.6. Garside structures had been called small Gaussian or thin Gaussian in earlier works until 2000 [16, 36-38].

Lemma 2.7. [15] Assume that $M$ is a Garside monoid. Then the following identities hold in $M$ :

$$
\begin{gathered}
(a b) \vee(a c)=a(b \vee c), \\
c \backslash(a b)=(c \backslash a)((a \backslash c) \backslash b), \quad(a b) \backslash c=b \backslash(a \backslash c), \\
(a \vee b) \backslash c=(a \backslash b) \backslash(a \backslash c)=(b \backslash a) \backslash(b \backslash c), \quad c \backslash(a \vee b)=(c \backslash a) \vee(c \backslash b) .
\end{gathered}
$$

Lemma 2.8. [15] Assume that $M$ is a Garside monoid. The following equivalent assertions hold : (i) There exists a mapping $\vee$ from $M$ to the integers satisfying $\vee(a)>0$ for every $a \neq 1$ in $M$, and $v(a b) \geq v(a)+v(b)$ for any $a, b$ in $M$;

(ii) For every set $X$ that generates $M$ and for every a in $M$, the lengths of the decompositions of a as products of elements in $X$ have a finite upper bound.

Definition 2.9. A monoid is said to be atomic if it satisfies the equivalent conditions of Lemma2.8. An atom is defined to be a nontrivial element $a$ such that $a=b c$ implies $b=1$ or $c=1$. The norm function $\|$.$\| of an atomic monoid M$ is defined in such a way that, for every $a$ in $M$, $\|a\|$ is the upper bound of the lengths of the decompositions of $a$ as products of atoms.

Example 2.10. The monoid $M_{\kappa}$ defined by the presentation

$$
\langle\mathrm{x}, \mathrm{y}: \mathrm{xyxyx}=\mathrm{yy}\rangle^{+}
$$

is another example of a Garside monoid, which admits no additive norm, i.e., no norm $v$ satisfying $v(a b)=v(a)+v(b)$ for any two elements $a, b$ in $M_{\mathrm{K}}$. Its group of fractions happens to be isomorphic to $\left\langle\mathrm{y}, \mathrm{z}: \mathrm{y}^{3}=\mathrm{z}^{3}\right\rangle$. See [36] for further details about $M_{\mathrm{K}}$.

By the previous lemma, every element in a Garside monoid has finitely many left-divisors, only then, for every pair of elements $(a, b)$, the common left-divisors of $a$ and $b$ admit a right-lcm, which is therefore the left-gcd of $a$ and $b$. This left-gcd will be denoted by $a \wedge b$. We define the right-gcd $\widetilde{\wedge}$ symmetrically.

Lemma 2.11. [15] Assume that $M$ is a Garside monoid. Then it admits a finite generating subset that is closed under $\backslash, /, \vee, \wedge, \widetilde{\vee}$, and $\widetilde{\wedge}$.

Every Garside monoid admits a finite set of atoms, and this set is the minimal generating set [16]. The hypothesis that there exists a finite generating subset that is closed under \implies that the closure of the atoms under $\backslash$ is finite.

Definition 2.12. If $M$ is a Garside monoid, the closure $S$ of its atoms under $\backslash$ and $\vee$ is finite-its elements are called simple elements, and their right-lcm is the (minimal) Garside element denoted by $\Delta$. The finite lattice $(S, \wedge, \vee, 1, \Delta)$ nicely summarises the whole structure of $M$ in a nutshell.

Definition 2.13. The set of all Garside elements of $M$ is $\Delta^{+}=\left\{\Delta^{p}: p>0\right\}$ (see [37] for instance) and we denote by $\Delta^{\frac{p}{q}}$ the set of the $q$-th roots of the element $\Delta^{p}$. For any root $h$ of a Garside 


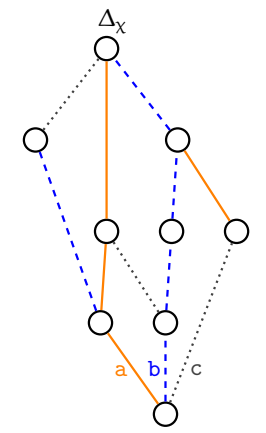

(a) The 9-simple lattice of $M_{\chi}$.

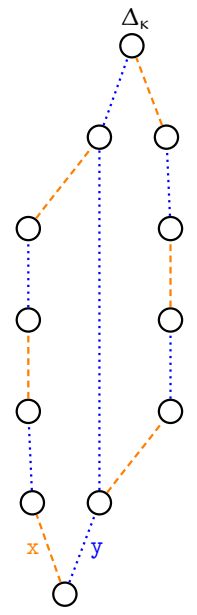

(b) The 12-simple lattice of $M_{\mathrm{K}}$.

FigurE 1. Two examples of lattices of simples from Examples 2.5 and 2.10.

element, we denote by $(\delta(h), \epsilon(h))$ the lexicographically minimal pair $(p, q)$ satisfying $h \in \Delta^{\frac{e p}{q}}$, where $e$ is the smallest positive integer for which $\Delta^{e}$ is central, hence $e=\epsilon(\Delta)$. In particular, the so-called exponent $\epsilon(h)$ of such a root $h$ is the smallest integer $q$ such that $h^{q}$ is central.

Example 2.14. The lattices of simple elements in $M_{\chi}$ of Example 2.5 and in $M_{\mathrm{K}}$ of Example 2.10 are displayed in Figures 1(a) and 1(b) More lattices are displayed in Figures 4, 6, and 8, Examples 3.8 and 4.5 will include the computation of some small sets $\Delta^{\frac{p}{q}}$ for $M_{\chi}$ and for $M_{\mathrm{K}}$.

Any Garside group can be the enveloping group of various monoids, many of whom can be Garside monoids. Now its centre gives rise to common structural constraints on all of them.

Lemma 2.15. [37] The centre of the group of fractions of a Garside monoid $M$ is the group of fractions of the centre of $M$.

Theorem 2.16. [37] The quasi-centre $Q Z$ (resp. the centre) of a Garside monoid $M$ is a free abelian submonoid of $M$, and the function $a \mapsto \Delta_{a}=\bigvee M \backslash a$ is a surjective semilattice homomorphism from $(M, \vee)$ onto $(Q Z, \vee)$.

Corollary 2.17. The free abelian group of rank $n$ is the group of fractions of a unique Garside monoid up to isomorphism.

We conclude this section by recalling how to effectively recognise Garside monoids.

Definition 2.18. A monoid presentation $\langle A: R\rangle^{+}$is called right-complemented if $R$ contains no $\varepsilon$-relation (that is, no relation $w=\varepsilon$ with $w$ nonempty), no relation $s \cdots=s \cdots$ with $s \in A$ and, for $s \neq t \in A$, at most one relation $s \cdots=t \cdots$.

Definition 2.19. A syntactic right-complement on an alphabet $A$ is a partial map $\theta$ from $A^{2}$ to $A^{*}$ such that $\theta(x, x)=\varepsilon$ holds for every $x \in A$ and, if $\theta(x, y)$ is defined, then so is $\theta(y, x)$. 
Lemma 2.20. [15] A monoid presentation $\langle A: R\rangle^{+}$is right-complemented if and only if there exists a syntactic right-complement $\theta$ such that $R$ consists of all relations $x \theta(x, y)=y \theta(y, x)$ with $x \neq y \in A$.

In the situation of Lemma 2.20, we naturally say that the presentation $\langle A: R\rangle^{+}$is associated with the syntactic right-complement $\theta$ (which is uniquely determined by the presentation), and then we write $\left\langle A: R_{\theta}\right\rangle^{+}$.

Lemma 2.21. [15] Assume that $\left\langle A: R_{\theta}\right\rangle^{+}$is a right-complemented presentation. Then there exists a unique minimal extension of the syntactic right-complement $\theta$ into a partial map $\theta$ from $A^{*} \times A^{*}$ to $A^{*}$ that satisfies the rules

$$
\begin{gathered}
\theta(u, \varepsilon)=\varepsilon, \quad \theta(\varepsilon, u)=u, \quad \theta(u v, u w)=\theta(v, w), \\
\theta(u, v w)=\theta(u, v) \theta(\theta(v, u), w), \quad \text { and } \quad \theta(v w, u)=\theta(w, \theta(v, u)) .
\end{gathered}
$$

The map $\theta$ is such that $\theta(u, v)$ exists if and only if $\theta(v, u)$ does.

Definition 2.22. Assume that $\left\langle A: R_{\theta}\right\rangle^{+}$is a right-complemented presentation. A triple $(u, v, w)$ of words in $A^{*}$ satisfies the $\theta$-cube condition whenever it satisfies

$$
\theta(\theta(u, v), \theta(u, w))=\theta(\theta(v, u), \theta(v, w)),
$$

meaning that either both sides are defined and they are equal, or neither is defined (see Figure 2). The $\theta$-cube condition is satisfied on $S \subseteq A^{*}$ if every triple of words in $S$ satisfies it.

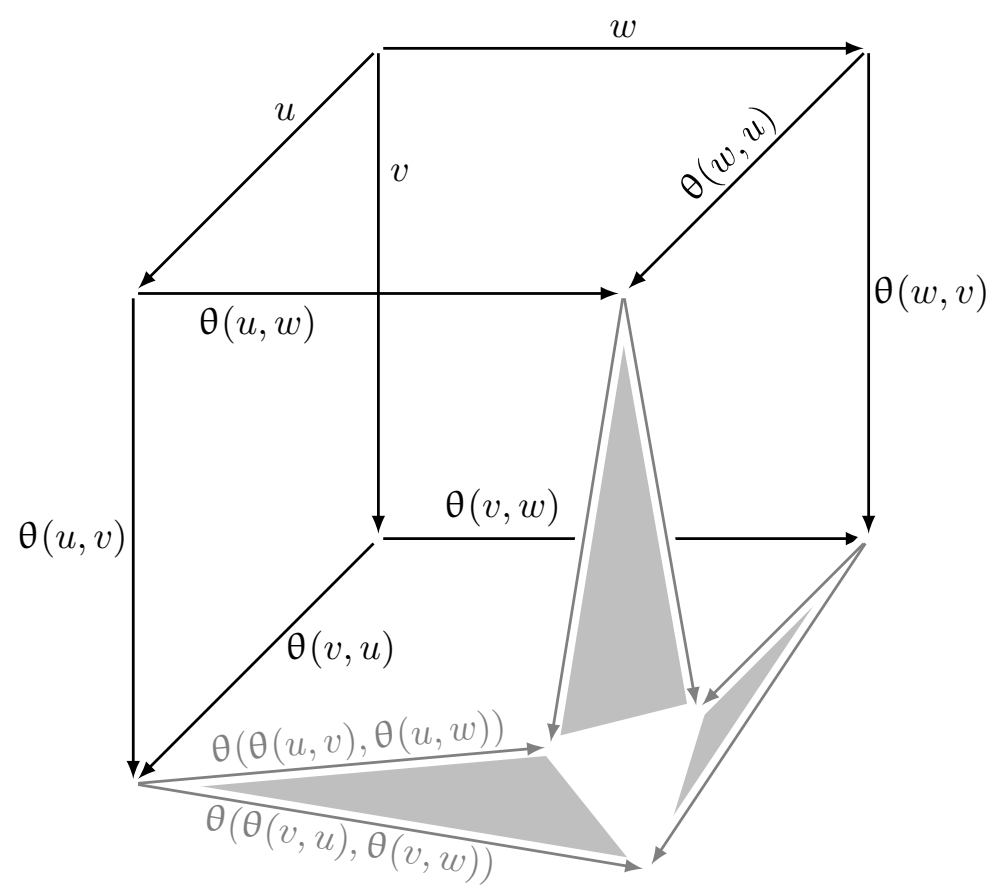

FIGURE 2. The $\theta$-cube condition: when one can draw the six faces of the cube, then each of the three small gray triangular sectors is labelled by two equal words, and the cube closes. See Definition 2.22. 
Rules $(\theta$-epsilon and ( $\theta$-extension $)$, and Condition $(\theta$-cube on words have to be compared with those of Lemma 2.7 on elements: any Garside monoid with set of atoms $A$ admits a rightcomplemented presentation $\left\langle A: R_{\theta}\right\rangle^{+}$satisfying Condition (0-cube) on $A^{*}$.

Example 2.23. The monoid $M_{\chi}$ from Example 2.5 admits the right-complemented presentation $\left\langle A: R_{\theta_{\chi}}\right\rangle^{+}$with $\theta_{\chi}(\mathrm{a}, \mathrm{b})=\mathrm{a}, \theta_{\chi}(\mathrm{a}, \mathrm{c})=\mathrm{a}^{2}, \theta_{\chi}(\mathrm{b}, \mathrm{a})=\mathrm{c}, \theta_{\chi}(\mathrm{b}, \mathrm{c})=\mathrm{b}^{2}, \theta_{\chi}(\mathrm{c}, \mathrm{a})=\mathrm{ab}$, and $\theta_{\chi}(\mathrm{c}, \mathrm{b})=\mathrm{a}$ (one could choose $\theta_{\chi}^{\prime}$ with $\theta_{\chi}^{\prime}(\mathrm{a}, \mathrm{c})=\mathrm{bc}$ instead). One can compute for instance $\theta_{\chi}\left(\theta_{\chi}(\mathrm{a}, \mathrm{b}), \theta_{\chi}(\mathrm{a}, \mathrm{cb})\right)=\theta_{\chi}(\mathrm{a}, \mathrm{aac})=\mathrm{ac}=\theta_{\chi}(\mathrm{c}, \mathrm{bba})=\theta_{\chi}\left(\theta_{\chi}(\mathrm{b}, \mathrm{a}), \theta_{\chi}(\mathrm{b}, \mathrm{cb})\right)$, witnessing the $\theta_{\chi}$-cube condition for the triple $(a, b, c b)$.

The criterion we shall use in the sequel is :

Theorem 2.24. [15] Assume that an atomic monoid $M$ admits a right-complemented presentation $\left\langle A: R_{\theta}\right\rangle^{+}$satisfying the $\theta$-cube condition on $A$. Then $M$ is left-cancellative and admits conditional right-lcms, that is, any two elements of $M$ that admit a common right-multiple admit a right-lcm.

Moreover, if $M$ is also right-cancellative and admits a Garside element, then $M$ is a Garside monoid. For alternative Garsidity criteria and details, we refer to [15] (see also [12,-14, 16]).

\section{Amalgamated FREE PRODUCTS}

We prove that the class of Garside groups is closed under some free products with cyclic amalgamated subgroups. This turns out to be exactly what we need to deduce that every tree product of infinite cyclic groups is a Garside group.

Definition 3.1. Let $M_{1}, M_{2}, H$ be monoids with morphisms $\phi_{1}: H \hookrightarrow M_{1}$ and $\phi_{2}: H \hookrightarrow M_{2}$. The amalgamated free product of $M_{1}$ and $M_{2}$ with respect to $H, \phi_{1}$, and $\phi_{2}$ is the monoid

$$
\left\langle M_{1} \star M_{2}: \phi_{1}(h)=\phi_{2}(h), h \in H\right\rangle^{+} .
$$

When $H=\langle h\rangle^{+}$is cyclic, we simply write $\phi_{1}(h)=h_{1}, \phi_{2}(h)=h_{2}$, and the amalgamated free product is denoted by $M_{1} \star_{h_{1}}=h_{2} M_{2}$.

Theorem 3.2. Let $M_{1}$ and $M_{2}$ be some Garside monoids. Then, for any root $h_{1}$ of any Garside element in $M_{1}$ and any root $h_{2}$ of any Garside element in $M_{2}$, the cyclic amalgamated free product $M_{1} \star_{h_{1}=h_{2}} M_{2}$ is a Garside monoid.

Actually, a necessary assumption is that $\phi_{i}(H)$ has to contain a Garside element of $M_{i}$ for $i \in$ $\{1,2\}$. When restricted to cyclic amalgamated submonoids, the latter naturally expresses in terms of roots of Garside elements.

Remark 3.3. Some algorithms for root extraction in Garside groups have been proposed in [29, 48, 49] : the extraction problem of an $n$-th root in a Garside group $G$ reduces to a conjugacy problem in the Garside group $\mathbb{Z} \ltimes G^{n}$ and hence is decidable (see [37,38]). Let us mention that the number of integers $n$ for which an element admits an $n$-th root is finite and that the number of conjugacy classes of the $n$-th roots of an element is finite.

Proof of Theorem 3.2 First, by hypothesis, the monoids $M_{1}$ and $M_{2}$ are cancellative and the amalgamated monoid $H$ is the (infinite) cyclic monoid, hence $\phi_{i}(H)=\left\langle h_{i}\right\rangle^{+}$is a so-called 


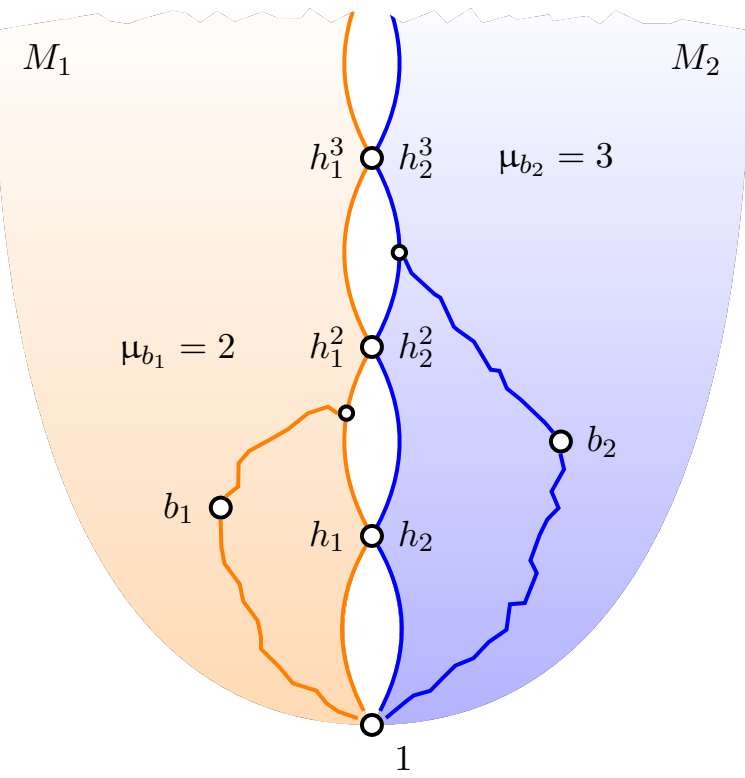

FIGURE 3. The lattice structure of the restriction to $M_{1} \vee M_{2}$ of the amalgamated free product $M_{1} \star_{h_{1}=h_{2}} M_{2}$. See the proof of Theorem 3.2.

unitary submonoid of $M_{i}$ for each $i \in\{1,2\}$, that is, either $h b \in \phi_{i}(H)$ or $b h \in \phi_{i}(H)$ together with $h \in \phi_{i}(H)$ imply $b \in \phi_{i}(H)$. According to [25, Corollary 3.4] (see also [17]), the monoids $M_{1}$ and $M_{2}$ both embed into the cyclic amalgamated product $M_{1} \star_{h_{1}=h_{2}} M_{2}$.

Next, the root assumption guarantees $M$ to inherit the atomicity from $M_{1}$ and $M_{2}$. Indeed, the lengths of the decompositions of any element $b \in M$ as products of atoms can be shown to be upper-bounded by the length of some central Garside element common to $M_{1}$, to $M_{2}$, and to $H$. Formally, by denoting $S_{j}=M_{j} \backslash h_{j} M_{j}$ for $j \in\{1,2\}$, it is known that the natural map $\psi_{j}: \phi_{j}(H) \times S_{j} \rightarrow M_{j}$ is bijective (see [18] for instance). We deduce that any element $b \in M$ admits a unique decomposition $h_{1}^{p} b_{1} b_{2} \cdots b_{2 n}$ with $b_{2 k-1} \in S_{1}, b_{2 k} \in S_{2}$, and $1 \leq k \leq n$. Now, for $j \in\{1,2\}$, each element $b_{2 k-j} \in S_{j}$ admits a minimal right-multiple of the form $b_{2 k-j} b_{2 k-j}^{\prime}=$ $h_{j}^{\epsilon\left(h_{j}\right) f_{2 k-j}}$ for some $b_{2 k-j}^{\prime} \in M_{j}$ and some integer $f_{2 k-j}>0$. Therefore we obtain

$$
b b_{2 n}^{\prime} \cdots b_{2}^{\prime} b_{1}^{\prime}=h_{1}^{p} h_{2}^{\epsilon\left(h_{2}\right) f_{2 n}} \cdots h_{2}^{\epsilon\left(h_{2}\right) f_{2}} h_{1}^{\epsilon\left(h_{1}\right) f_{1}}=h_{1}^{N_{b}}=h_{2}^{N_{b}},
$$

where $N_{b}$ is the uniquely determined number $p+\epsilon\left(h_{1}\right) \sum_{k=1}^{n} f_{2 k-1}+\epsilon\left(h_{2}\right) \sum_{k=1}^{n} f_{2 k}$. Denoting by $\|\cdot\|_{1},\|\cdot\|_{2}$, and $\|\cdot\|$ the norms of $M_{1}, M_{2}$, and $M$, respectively, we conclude

$$
\|b\| \leq \max \left(\left\|h_{1}^{N_{b}}\right\|_{1},\left\|h_{2}^{N_{b}}\right\|_{2}\right)
$$

which gives the claim.

Let $A_{i}$ be the set of atoms of $M_{i}$ and let $\theta_{i}$ be a syntactic right-complement such that $M_{i}$ admits the complemented presentation $\left\langle A_{i}: R_{\theta_{i}}\right\rangle^{+}$for $i \in\{1,2\}$. By definition, the cyclic amalgamated free product monoid $M=M_{1} \star h_{1}=h_{2} M_{2}$ admits the presentation

$$
\langle A: R\rangle^{+} \quad \text { with } \quad A=A_{1} \sqcup A_{2} \quad \text { and } \quad R=R_{\theta_{1}} \sqcup R_{\theta_{2}} \sqcup\left\{u_{1}=u_{2}\right\} \text {, }
$$


where $u_{i} \in A_{i}^{*}$ is any fixed representative of $h_{i}$ for $i \in\{1,2\}$.

We shall prove that $M$ admits a complemented presentation $\left\langle A: R_{\theta}\right\rangle^{+}$where the syntactic rightcomplement $\theta$ essentially extends the syntactic right-complements $\theta_{1}$ and $\theta_{2}$. Formally, we first simply set

$$
\theta(x, y)=\theta_{i}(x, y) \quad \text { for } \quad(x, y) \in A_{i}^{2} \quad \text { and } \quad i \in\{1,2\} .
$$

For $w \in A_{i}^{*}$ and $i \in\{1,2\}$, the root $h_{i}$ admits powers which are right-multiples of the element represented by $w$, so one can define $\mu_{w}=\min \left\{m: \theta_{i}\left(u_{i}^{m}, w\right)=\varepsilon\right\}$ (see Figure 3). Therefore we set:

$$
\theta(x, y)=\left\{\begin{array}{ll}
\theta_{i}\left(x, u_{i}^{\mu_{y}}\right) & \text { for } \mu_{x} \geq \mu_{y}, \\
\theta_{i}\left(x, u_{i}^{\mu_{x}}\right) \theta_{3-i}\left(u_{3-i}^{\mu_{x}}, y\right) & \text { for } \mu_{x}<\mu_{y},
\end{array} \quad \text { for } \begin{array}{rl}
(x, y) & \in A_{i} \times A_{3-i}, \\
i & \in\{1,2\} .
\end{array}\right.
$$

By construction, $R_{\theta}$ includes $R$. In particular, for any atom $x_{i} \in A_{i}$ left-dividing $u_{i}$ for $i \in\{1,2\}$, we have $\mu_{x_{i}}=1$ and $\theta\left(x_{i}, x_{3-i}\right)=\theta_{i}\left(x_{i}, u_{i}\right)$, hence $\left\{u_{1}=u_{2}\right\} \subseteq R_{\theta}$. Conversely, any relation $x \theta(x, y)=y \theta(y, x)$ in $R_{\theta}$ derives from relations in $R$. Indeed, for $(x, y) \in A_{i} \times A_{3-i}$ and say $\mu_{x}<\mu_{y}$, we have:

$$
\begin{array}{rll}
x \theta(x, y) & \stackrel{\text { def }}{=} & x \theta_{i}\left(x, u_{i}^{\mu_{x}}\right) \theta_{3-i}\left(u_{3-i}^{\mu_{x}}, y\right) \\
& \equiv_{\theta_{i}} & u_{i}^{\mu_{x}} \theta_{3-i}\left(u_{3-i}^{\mu_{x}}, y\right) \\
& \equiv & u_{3-i}^{\mu_{x}} \theta_{3-i}\left(u_{3-i}^{\mu_{x}}, y\right) \\
& \equiv_{\theta_{3-i}} & y \theta_{3-i}\left(y, u_{3-i}^{\mu_{x}}\right) \\
& \stackrel{\text { def }}{=} & y \theta(y, x) .
\end{array}
$$

The case $\mu_{x}=\mu_{y}$ is even simpler:

$$
x \theta(x, y) \stackrel{\text { def }}{=} x \theta_{i}\left(x, u_{i}^{\mu_{y}}\right) \equiv_{\theta_{i}} u_{i}^{\mu_{x}} \equiv u_{3-i}^{\mu_{x}} \equiv_{\theta_{3-i}} y \theta_{3-i}\left(y, u_{3-i}^{\mu_{x}}\right) \stackrel{\text { def }}{=} y \theta(y, x) .
$$

So $\theta$ is well-defined and gives to $M$ a right-complemented presentation $\left\langle A: R_{\theta}\right\rangle^{+}$.

The syntactic right-complement $\theta$ is defined on $A^{2}$ and, by Lemma 2.21, it can be uniquely extended by using

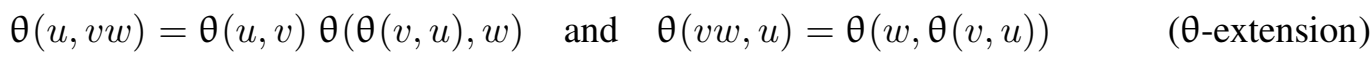

for any $u, v, w \in A^{*}$.

The point is now to check the $\theta$-cube condition. Since $M$ is atomic, we only need to check it on $A$. It suffices to take say $(x, z) \in A_{1}^{2}$ and $y \in A_{2}$, since the other cases are either symmetric or trivial. To make reading easier, $X_{i}, Y_{i}$, and $Z_{i}$ will denote $u_{i}^{\mu_{x}}, u_{i}^{\mu_{y}}$, and $u_{i}^{\mu_{z}}$ respectively for $i \in\{1,2\}$. For $\mu_{x}>\mu_{z}>\mu_{y}$, we have

$$
\begin{aligned}
& \left\{\begin{aligned}
\theta(\theta(x, y), \theta(x, z)) & =\theta_{1}\left(\theta_{1}\left(x, Y_{1}\right), \theta_{1}(x, z)\right) & \\
& =\theta_{1}\left(\theta_{1}\left(Y_{1}, x\right), \theta_{1}\left(Y_{1}, z\right)\right), & \text { ( } \theta_{1} \text {-cube) } \\
\theta(\theta(y, x), \theta(y, z)) & =\theta\left(\theta_{2}\left(y, Y_{2}\right) \theta_{1}\left(Y_{1}, x\right), \theta_{2}\left(y, Y_{2}\right) \theta_{1}\left(Y_{1}, z\right)\right) & \\
& =\theta_{1}\left(\theta_{1}\left(Y_{1}, x\right), \theta_{1}\left(Y_{1}, z\right)\right), & \text { ( } \theta \text {-epsilon) }
\end{aligned}\right. \\
& \left\{\begin{aligned}
\theta(\theta(z, y), \theta(z, x)) & =\theta_{1}\left(\theta_{1}\left(z, Y_{1}\right), \theta_{1}(z, x)\right) & \\
& =\theta_{1}\left(\theta_{1}\left(Y_{1}, z\right), \theta_{1}\left(Y_{1}, x\right)\right), & \text { ( } \theta_{1} \text {-cube) }
\end{aligned}\right. \\
& \begin{aligned}
\theta(\theta(y, z), \theta(y, x)) & =\theta\left(\theta_{2}\left(y, Y_{2}\right) \theta_{1}\left(Y_{1}, z\right), \theta_{2}\left(y, Y_{2}\right) \theta_{1}\left(Y_{1}, x\right)\right) \\
& =\theta_{1}\left(\theta_{1}\left(Y_{1}, z\right), \theta_{1}\left(Y_{1}, x\right)\right) .
\end{aligned}
\end{aligned}
$$


For $\mu_{x}>\mu_{y}>\mu_{z}$, we have

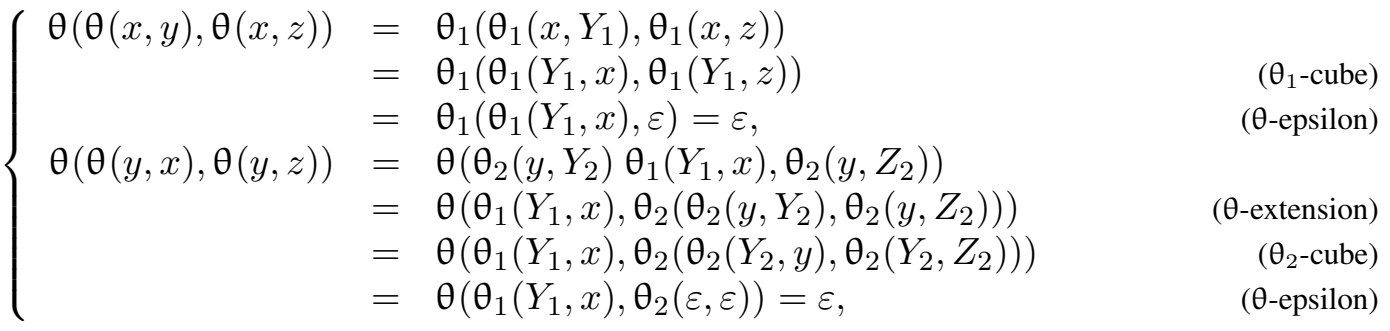

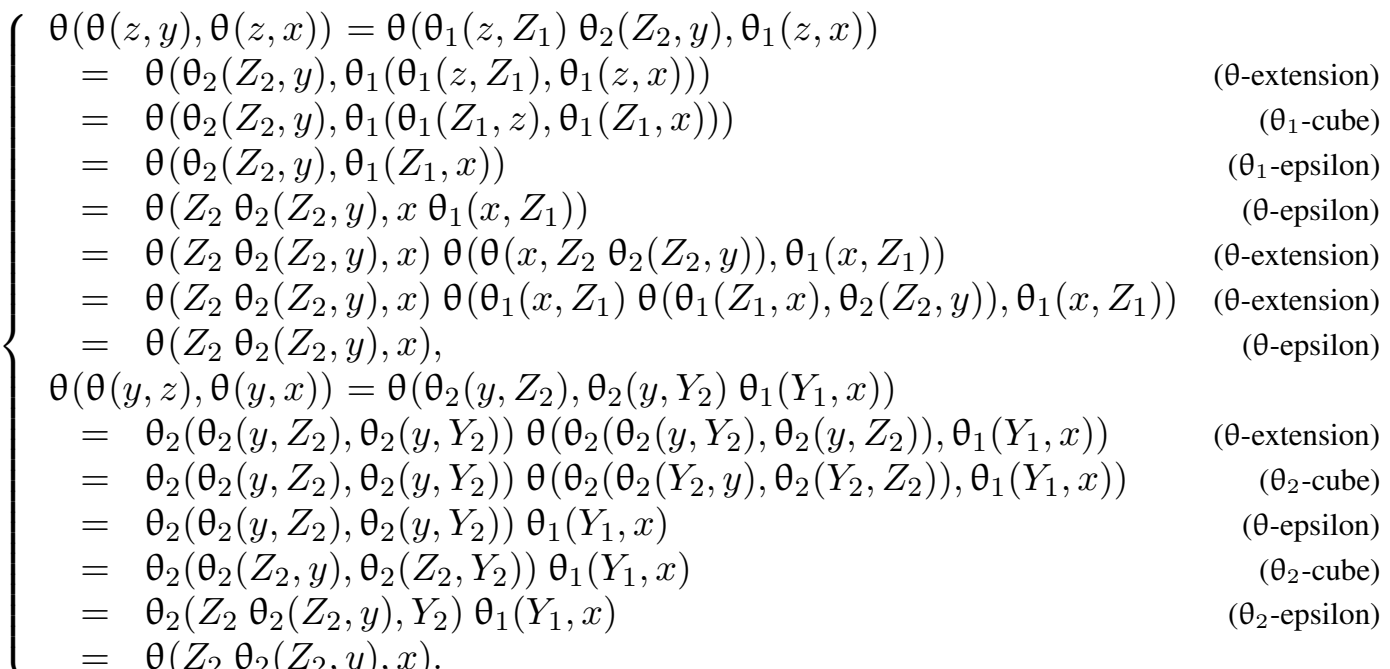

For $\mu_{y}>\mu_{x}>\mu_{z}$, we have

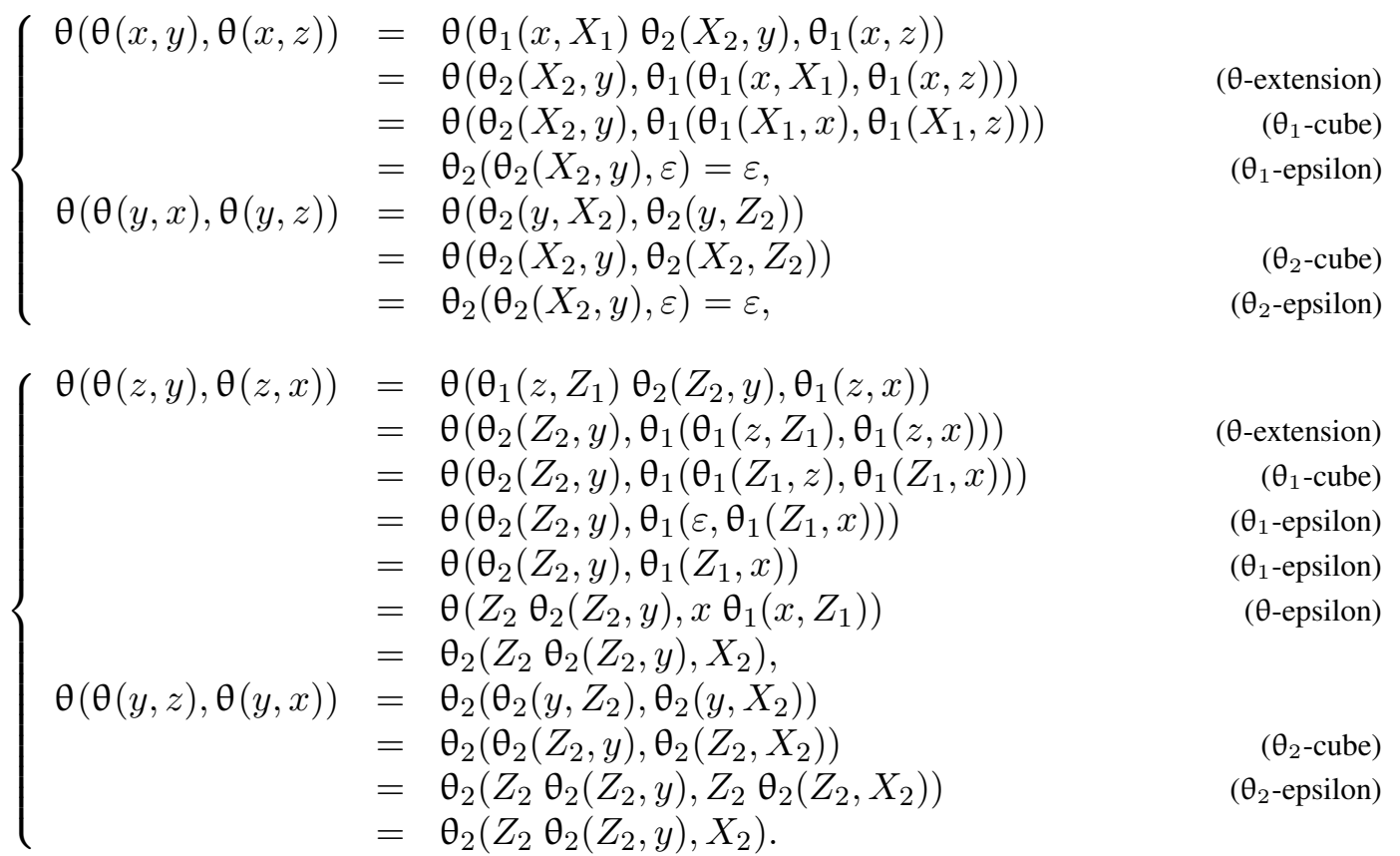


This completes the proof that the syntactic right-complement associated with $\left\langle A: R_{\theta}\right\rangle^{+}$satisfies the $\theta$-cube condition. By Theorem [2.24, we deduce that the amalgamated free product $M=$ $M_{1} \star h_{1}=h_{2} M_{2}$ is left-cancellative and admits conditional right-lcms. A symmetric argument allows to conclude that $M$ is cancellative and admits both left- and right-conditional lcms.

Finally, let $\Delta_{i}$ denote the smallest Garside element in $M_{i}$ of which $h_{i}$ is a root, say $\Delta_{i}=h_{i}^{p_{i}}$ for $i \in\{1,2\}$. We naturally define $\Delta=h^{p_{1} \vee p_{2}}$ with $h=h_{1}=h_{2}$. By construction, $\Delta$ inherits the quasi-centrality from $\Delta_{1}$ and $\Delta_{2}$ : for any $b \in M$, there exists an element $b^{\prime} \in M$ satisfying $\Delta b=b^{\prime} \Delta$. Therefore, for any left-divisor $d$ of $\Delta$, say $\Delta=d b$, we find $\Delta b=b^{\prime} \Delta=b^{\prime} d b$, hence $\Delta=b^{\prime} d$ by right-cancellativity. Using a symmetric argument, we deduce that the set of its right-divisors coincides with the set of its left-divisors. The latter includes $A_{1} \sqcup A_{2}$ by definition, thus generates $M$. Therefore, $\Delta$ is a Garside element for $M$.

Remark 3.4. An extremal case-when the roots are chosen to correspond to some powers of minimal Garside elements - has been considered very early in [16, 36].

Remark 3.5. An amalgamated free product of cancellative monoids $M_{1}$ and $M_{2}$ along a (cancellative) monoid $H$ need not inherit the cancellativity of $M_{1}$ and $M_{2}$. While the unitarity assumption on $H$ ensures the embedding of both $M_{1}$ and $M_{2}$ into the amalgamated free product, it is not sufficient to guarantee the cancellativity of the latter (see for instance the counterexample of [26, Section 3]).

Remark 3.6. With different approaches and motivations, a related result appeared in the context of so-called preGarside monoids [23]. No condition for the existence of a Garside element is considered. A so-called special property on $H$ (stronger than unitarity) is required. The latter is far to be satisfied in our cyclic amalgam framework.

Example 3.7. The simplest examples are those monoids $K_{p, q}^{+}=\left\langle\mathrm{s}, \mathrm{t}: \mathrm{s}^{p}=\mathrm{t}^{q}\right\rangle^{+}$obtained with $M_{1}=\langle\mathrm{s}\rangle^{+}, M_{2}=\langle\mathrm{t}\rangle^{+}$, and $H=\langle\mathrm{h}\rangle^{+}$with $\phi_{1}: \mathrm{h} \mapsto \mathrm{s}^{p}$ and $\phi_{2}: \mathrm{h} \mapsto \mathrm{t}^{q}$. They are well-known Garside monoids associated with torus knot groups (whenever $p$ and $q$ are coprime), see [15, 16, 36, 40]. For a generalisation, see Corollary 3.10 and related Example 3.11 below.

Example 3.8. Take again $M_{\chi}=\left\langle\mathrm{a}, \mathrm{b}, \mathrm{c}: \mathrm{a}^{2}=\mathrm{bc}, \mathrm{b}^{3}=\mathrm{ca}\right\rangle^{+}$from Example 2.5 and $M_{\mathrm{k}}=$ $\langle\mathrm{x}, \mathrm{y}: \operatorname{xyxyx}=\mathrm{yy}\rangle^{+}$from Example 2.10. Choose the amalgamated submonoid $H=\langle\mathrm{h}\rangle^{+}$ with $\phi_{1}: \mathrm{h} \mapsto \mathrm{cb} \in \Delta_{\chi}^{\frac{2}{3}}$ and $\phi_{2}: \mathrm{h} \mapsto$ yxyx $\in \Delta_{\mathrm{K}}^{\frac{2}{3}}$ for instance. Then $M_{\chi} \star_{\mathrm{cb}=\mathrm{yxyx}} M_{\mathrm{K}}$ is a Garside monoid with minimal Garside element $b^{8}=(\mathrm{cb})^{3}=(\text { yxyx })^{3}$ admitting 161 simples, whose lattice is displayed in Figure 4. Table 1 further illustrates the wide variety of those Garside monoids obtained as free products with cyclic amalgamation $M_{\chi}{ }^{\star} h_{\chi}=h_{\mathrm{K}} M_{\mathrm{K}}$, even when one arbitrarily restricts the roots $h_{\chi}$ and $h_{\mathrm{K}}$ to be chosen respectively from the set $\Delta_{\chi}^{\frac{p}{q}}$ with, say, $\frac{p}{q} \in$ $\left\{\frac{1}{4}, \frac{1}{3}, \frac{1}{2}, \frac{2}{3}, \frac{1}{1}\right\}$ and the set $\Delta_{\kappa}^{\frac{p}{q}}$ with $\frac{p}{q}=\left\{\frac{1}{3}, \frac{1}{2}, \frac{1}{1}\right\}$. Such Garside structures may become huge: choosing $h_{\chi} \in \Delta_{\chi}^{\frac{3}{4}}$ for instance, we obtain almost 74 million simples for $M_{\chi} \star_{\text {ac }=\text { xxyxy }} M_{\mathrm{K}}$, and about seven times for $M_{\chi} \star_{\text {ca }=\text { xxyxy }} M_{\kappa}$.

From Theorem 3.2, we deduce the following corollary that establishes a complete characterisation of those cyclic amalgamated free products of Garside groups which are Garside as well. 


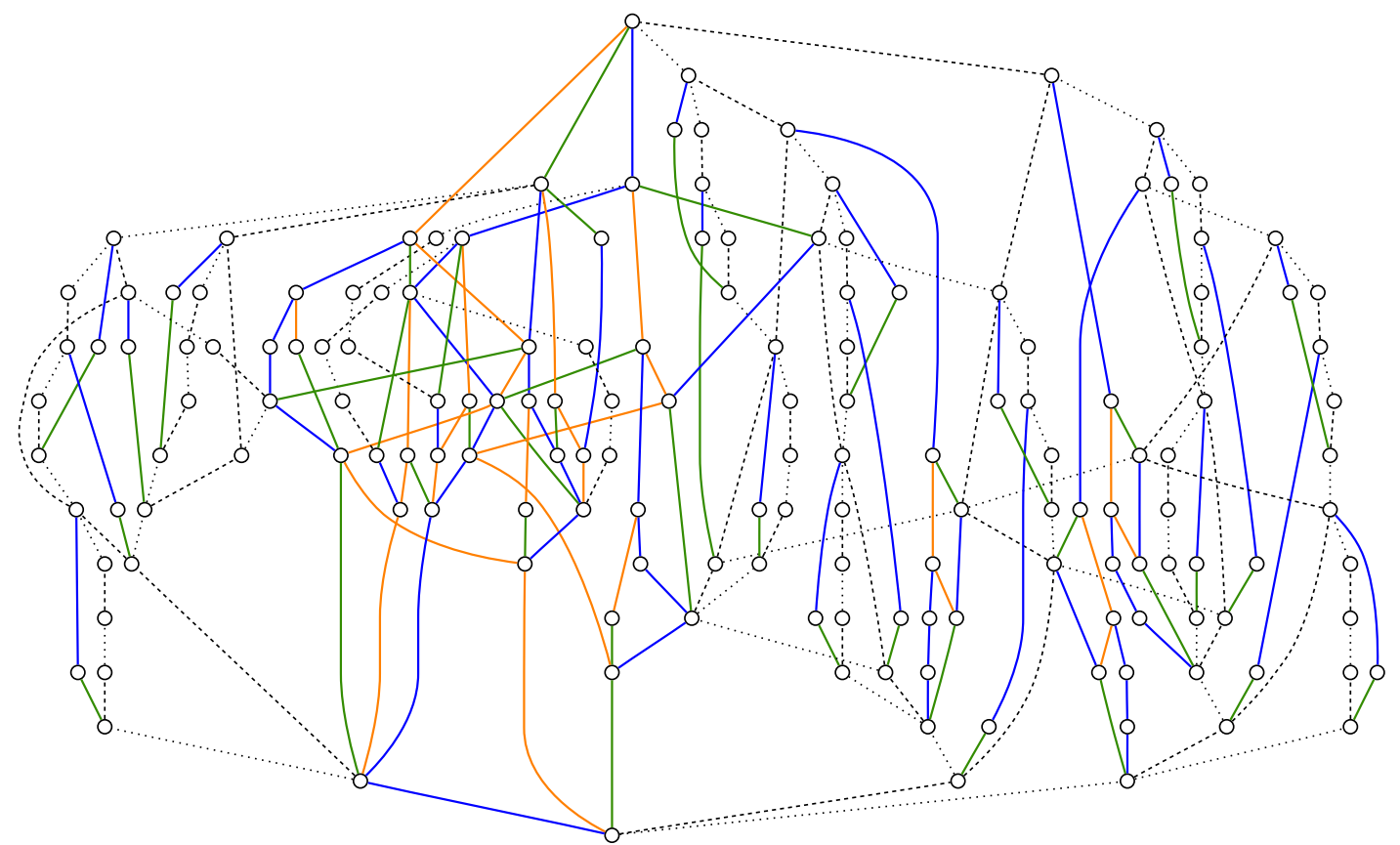

FIGURE 4. See Example 3.8, the 161-simple lattice of the amalgamated free product monoid $M_{\chi} \star_{\mathrm{cb}=\mathrm{yxyx}} M_{\mathrm{K}}$.

\begin{tabular}{|c|c|c|c|c|c|c|c|c|c|c|c|c|}
\hline & & \multicolumn{3}{|c|}{$\Delta_{\kappa}^{\frac{1}{3}}$} & \multicolumn{7}{|c|}{$\Delta_{K}^{\frac{2}{3}}$} & \multirow{2}{*}{$\frac{\Delta_{\kappa}^{\frac{1}{1}}}{\text { yуy }}$} \\
\hline & $h_{\chi}{ }^{h_{\kappa}}$ & $\mathrm{y}$ & xy & $\mathrm{yx}$ & xyxy & yxyx & yy & xxyxy & xyxxy & yxxyx & yxyxx & \\
\hline$\Delta_{\chi}^{\frac{1}{4}}$ & b & 4242 & 4611 & 4611 & 580609 & 580609 & 572541 & 510474 & 510474 & 510474 & 510474 & 1161 \\
\hline$\Delta_{x}^{\frac{1}{3}}$ & a & 17 & 19 & 19 & 74 & 74 & 78 & 76 & 76 & 76 & 76 & 301 \\
\hline$\Delta_{\chi}^{\frac{1}{2}}$ & $\mathrm{bb}$ & 858 & 760 & 760 & 12622 & 12622 & 11010 & 7904 & 7904 & 7904 & 7904 & 89 \\
\hline$\frac{2}{3}$ & aa & 71 & 70 & 70 & 249 & 249 & 253 & 206 & 206 & 206 & 206 & 831 \\
\hline & $\mathrm{cb}$ & 57 & 57 & 57 & 161 & 161 & 157 & 140 & 140 & 140 & 140 & 501 \\
\hline$\Delta_{\chi}^{\frac{1}{1}}$ & aaa & 300 & 225 & 225 & 917 & 917 & 837 & 611 & 611 & 611 & 611 & 19 \\
\hline
\end{tabular}

TABLE 1. See Example 3.8 the number of simples of the Garside monoid $M_{\chi} \star h_{\chi}=h_{\kappa} M_{\mathrm{K}}$ for roots $h_{\chi} \in \Delta_{\chi}^{\frac{p}{q}}$ with $\frac{p}{q} \in\left\{\frac{1}{4}, \frac{1}{3}, \frac{1}{2}, \frac{2}{3}, \frac{1}{1}\right\}$ and $h_{\mathrm{\kappa}} \in \Delta_{\mathrm{K}}^{\frac{p}{q}}$ with $\frac{p}{q}=\left\{\frac{1}{3}, \frac{1}{2}, \frac{1}{1}\right\}$. 
Corollary 3.9. Let $M_{1}$ and $M_{2}$ be some Garside monoids. The (enveloping group of) the cyclic amalgamated free product $M_{1} \star_{h_{1}=h_{2}} M_{2}$ is Garside if and only if $h_{1}$ is a root of some Garside element in $M_{1}$ and $h_{2}$ is a root of some Garside element in $M_{2}$.

Proof. Theorem 3.2 coincides with $(\Leftarrow)$, so it suffices to show $(\Rightarrow)$. The centre of the amalgamated free product $M=M_{1} \star_{h_{1}=h_{2}} M_{2}$ is $Z(M)=Z\left(M_{1}\right) \cap Z\left(M_{2}\right)$ (see [32] or [45] for instance). Since the amalgamated submonoid $H$ is infinite cyclic by hypothesis, $Z(M)$ is then either trivial or infinite cyclic. Now, since $M$ is nontrivial and assumed to be Garside, $Z(M)$ cannot be trivial, hence $Z(M)$ is infinite cyclic, say $\langle z\rangle^{+}$. Therefore, the central Garside element $z$ is some nontrivial power of $h_{i}$ for $i \in\{1,2\}$.

At this point we have to emphasise the associativity of the free product with cyclic amalgamated submonoids in Theorem 3.2. A consequence is the following corollary.

A weighted tree $\mathcal{T}$ is a tree with vertex set $V(\mathcal{T})$ and edge set $E(\mathcal{T})$ together with a weight map $\omega$ which, with every edge between two vertices $a$ and $b$, associates two nonzero integer weights $\omega_{a, b}$ and $\omega_{b, a}$. Such a double weighting is displayed by using label pairs: $a \frac{\omega_{a, b} \quad \omega_{b, a}}{b} b$. In this case, the tree product $G(\mathcal{T})$ is the group presented by

$$
\left\langle a \in V(\mathcal{T}): a^{\omega_{a, b}}=b^{\omega_{b, a}} \text { for }\{a, b\} \in E(\mathcal{T})\right\rangle .
$$

Corollary 3.10. Every tree product of infinite cyclic groups is a Garside group.

Example 3.11. Consider the weighted trees of Figure 5. The positively reduced tree $\mathcal{T}_{0}^{\prime}$ (right) has vertices with extra gray labels that will be explained later in the proof of Theorem 4.2 and in Example 4.9. According to Corollary 3.10, both trees present the same Garside group $G\left(\mathcal{T}_{0}\right)$.
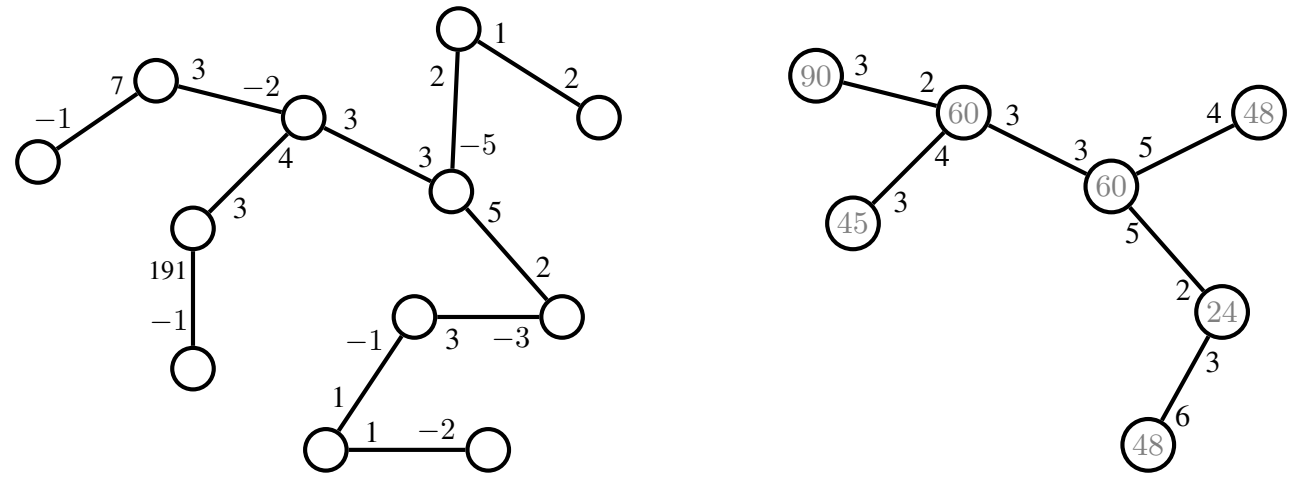

FIGURE 5. See Examples 3.11 and 4.9, a rather general weighted tree $\mathcal{T}_{0}$ (left) and the corresponding positively reduced weighted tree $\mathcal{T}_{0}^{\prime}$ (right); both generate the same Garside group $G\left(\mathcal{T}_{0}\right)$ by Corollary 3.10 .

Proof of Corollary 3.10 The point is to show that any tree with nonzero weights can be transformed into another-generating the same group — with weights all belonging to $\{2,3,4 \ldots\}$. Such a transformation actually requires two steps, say a positive transformation and an atomic transformation, which happen to be commutative.

The atomic transformation amounts to delete any vertex $a$ admitting a weight $\omega(a, b)= \pm 1$ for some vertex $b$ and, therefore, to connect the latter to each vertex $c$ among the other possible former 
neighbours of $a$ with the weights $\omega(c, b)=\omega(c, a)$ and $\omega(b, c)= \pm \omega(b, a) \omega(a, c)$. By applying a finite sequence of such so-called elementary collapses, we finally obtain a tree-generating the same group — without weight \pm 1 , which is well known as a reduced weighted tree (see [30] for instance).

Especially relevant for the tree case, the positive transformation ultimately amounts to simply take the absolute value of each weight, but it can be rigorously described and justified as follows. First root the tree at any distinguished vertex $v$. Then apply the following recursive algorithm from $v$ to the leaves:

- for each vertex $b \neq v$ with parent $a$, if $\omega_{b, a}$ is negative, then take the opposite of each weight $\omega_{b, c}$ with $c$ neighbour of $b$ (this corresponds with exchanging the generator $b$ into its inverse $b^{-1}$ );

- for each vertex $b$, for each child $c$ with $\omega_{b, c}$ negative, take the opposite of both $\omega_{b, c}$ and $\omega_{c, b}$ (this corresponds with rewriting the relation $b^{\omega_{b, c}}=c^{\omega_{c, b}}$ into $b^{-\omega_{b, c}}=c^{-\omega_{c, b}}$ ).

Therefore, from any finite weighted tree $\mathcal{T}$, we obtain a positively reduced weighted tree $\mathcal{T}^{\prime}$ - that is, whose weights are all in $\{2,3,4 \ldots\}$-satisfying $G(\mathcal{T}) \cong G\left(\mathcal{T}^{\prime}\right)$. Now, the monoid $G^{+}\left(\mathcal{T}^{\prime}\right)$ presented by $\left\langle a \in V\left(\mathcal{T}^{\prime}\right): a^{\omega_{a, b}}=b^{\omega_{b, a}} \text { for }\{a, b\} \in E\left(\mathcal{T}^{\prime}\right)\right\rangle^{+}$is an atomic monoid by Definition 2.9 and thus a Garside monoid by Theorem 3.2 and Corollary 3.9, that makes $G(\mathcal{T})$ a Garside group.

\section{HNN EXTENSIONS}

We characterise and study those cyclic HNN extensions of a Garside monoid whose enveloping groups are Garside as well (under some mild atomicity condition). Again the roots of Garside elements play a crucial role.

Definition 4.1. Let $M$ and $H$ be two monoids with morphisms $\phi_{1}: H \hookrightarrow M$ and $\phi_{2}: H \hookrightarrow M$. The $H N N$ extension of $M$ with respect to $H, \phi_{1}$, and $\phi_{2}$ is the monoid

$$
\left\langle M, t: \phi_{1}(h) t=t \phi_{2}(h), h \in H\right\rangle^{+} .
$$

Theorem 4.2. Let $M$ be a Garside monoid and $H$ be the infinite cyclic monoid $\langle h\rangle^{+}$with a morphism $\phi_{i}: H \hookrightarrow M$ for $i \in\{1,2\}$ satisfying $\left\|\phi_{1}(h)\right\|=\left\|\phi_{2}(h)\right\|$. Then the enveloping group of the HNN extension $\left\langle M, t: \phi_{1}(h) t=t \phi_{2}(h)\right\rangle^{+}$is a Garside group if and only if $\phi_{1}(h)$ and $\phi_{2}(h)$ are two $n$-th roots of the same Garside element in $M$ for some $n>0$.

Here again, a necessary assumption is actually that $\phi_{1}(H)$ and $\phi_{2}(H)$ have to contain the same Garside element of $M$. When restricted to cyclic HNN extensions, the latter naturally expresses in terms of roots of Garside elements (see also Remark 3.3).

Proof. Let $\bar{M}$ denote the HNN extension $\left\langle M, t: h_{1} t=t h_{2}\right\rangle^{+}$where $h_{i}$ denotes $\phi_{i}(h)$ for $i \in$ $\{1,2\}$ and let $\bar{G}$ denote its enveloping group $\left\langle G, t: h_{1} t=t h_{2}\right\rangle$.

By hypothesis, the monoid $M$ is cancellative and the monoid $H$ is the infinite cyclic monoid, hence $\phi_{i}(H)=\left\langle h_{i}\right\rangle^{+}$is a so-called unitary submonoid of $M$ for each $i \in\{1,2\}$ (see also the proof of Theorem 3.2). Therefore, according to [27, Theorem 1], the monoid $M$ embeds into its cyclic HNN extension $\bar{M}$.

$(\Rightarrow)$ The centre $Z(\bar{G})$ of $\bar{G}$ is the subgroup $Z(G) \cap \phi_{1}(K) \cap \phi_{2}(K)$ [32,45] where $K$ denotes the group of fractions of $H$ and the morphism $\phi_{i}: K \rightarrow G$ extends $\phi_{i}: H \rightarrow M$ for each $i \in\{1,2\}$. 
Since $K$ is infinite cyclic by hypothesis, $Z(\bar{G})$ is then either trivial or infinite cyclic. Now, since $\bar{G}$ is nontrivial and assumed to be Garside, $Z(\bar{G})$ cannot be trivial, hence $Z(\bar{G})$ is infinite cyclic.

By Corollary 2.17, $Z(\bar{G})$ is the group of fractions of some $\langle z\rangle^{+}=Z(M) \cap \phi_{1}(H) \cap \phi_{2}(H)$. Therefore, the Garside element $z$ is some nontrivial power $h_{1}^{\ell}$ of $h_{1}$ which has to be central in $G$.

Moreover, $h_{1}^{\ell}$ has to commute with $t$, that is, $h_{1}^{\ell} t=t h_{1}^{\ell}$ holds. Now, $h_{1}$ satisfies $h_{1} t=t h_{2}$ by definition of $\bar{G}$, hence $h_{1}^{d} t=t h_{2}^{d}$ for any $d \in \mathbb{Z}$. We find $t h_{1}^{\ell}=t h_{2}^{\ell}$ in $\bar{G}$, hence $h_{1}^{\ell}=h_{2}^{\ell}$ in $Z(G)$.

$(\Leftarrow)$ Let $A$ be the set of atoms of $M$. As it stands, the monoid $\bar{M}$ need not provide a Garside structure to its enveloping group $\bar{G}$. The trick is to introduce the map $\psi$ defined by

$$
\psi: x \mapsto \begin{cases}t & \text { for } x=t \\ x t & \text { for } x \in A .\end{cases}
$$

which uniquely extends to a group isomorphism, corresponding to a change of generators. We claim that the submonoid of $\psi(\bar{M})$ generated by $A \sqcup\{t\}$ is Garside. We consider the natural candidate-monoid $\overline{\bar{M}}$ whose presentation is obtained from that of $\bar{M}$ by keeping the set of atoms $A \sqcup\{t\}$ and by stuffing each relation with the stable atom $t$. Formally, for any nonempty word $u=\prod_{i=1}^{m} a_{i}$ with $a_{i} \in A$, the word denoted by $\bar{u}^{t}$ is $a_{1} \prod_{i=2}^{m}\left(t a_{i}\right)$. So, every relation $u=v$ from $M$ becomes $\bar{u}^{t}=\bar{v}^{t}$ in $\overline{\bar{M}}$ and the special relation $h_{1} t=t h_{2}$ becomes $\overleftarrow{h}_{1}^{t} t=t \bar{h}_{2}^{t}$ in $\overline{\bar{M}}$. We shall show that $\overline{\bar{M}}$ is a Garside monoid whose enveloping group is the HNN extension $\bar{G}$.

First, the assumption $\left\|\phi_{1}(h)\right\|=\left\|\phi_{2}(h)\right\|$ guarantees $\overline{\bar{M}}$ to inherit the atomicity from $M$. Indeed, the mapping $\overline{\bar{v}}$ from $\overline{\bar{M}}$ to the integers defined by

$$
\overline{\bar{v}}\left(b_{0} t b_{1} t \cdots t b_{n}\right)=n+\sum_{k=0}^{n}\left\|b_{k}\right\|
$$

with $b_{k} \in M$ for $0 \leq k \leq n$ satisfies Condition (i) from Lemma 2.8 (the same holds for $\bar{M}$ ).

Let $M=\left\langle A: R_{\theta}\right\rangle^{+}$. By definition, we have

$$
\bar{M}=\left\langle A \sqcup\{t\}: R_{\theta} \sqcup\left\{u_{1} t=t u_{2}\right\}\right\rangle^{+},
$$

where $u_{i} \in A^{*}$ is any fixed representative of $h_{i}$ for $i \in\{1,2\}$. We deduce

$$
\overline{\bar{M}}=\left\langle A \sqcup\{t\}: \bigsqcup_{(x, y) \in A^{2}}\left\{\overline{x \theta(x, y)}^{t}=\overline{y \theta(y, x)}^{t}\right\} \sqcup\left\{{\overline{u_{1}}}^{t} t=t{\overline{u_{2}}}^{t}\right\}\right\rangle^{+} .
$$

We shall prove that $\overline{\bar{M}}$ admits a complemented presentation $\left\langle A \sqcup\{t\}: R_{\bar{\theta}}\right\rangle^{+}$where the syntactic right-complement $\overline{\bar{\theta}}$ essentially extends the syntactic right-complement $\theta$ via the map $\psi$. Formally, we first simply set

$$
\overline{\bar{\theta}}(x, y)=t \overline{\theta(x, y)}^{t} \quad \text { for } \quad(x, y) \in A^{2} .
$$

Let $x_{1} \in A$ denote the leftmost letter of $u_{1}$ and let $v_{1} \in A^{*}$ be the word satisfying $u_{1}=x_{1} v_{1}$. Then we set:

$$
\overline{\bar{\theta}}\left(x_{1}, t\right)=t{\bar{v}_{1}}^{t} t \quad \text { and } \quad \overline{\bar{\theta}}\left(t, x_{1}\right)={\overline{u_{2}}}^{t} .
$$

Finally, for $x \in A \backslash\left\{x_{1}\right\}$, we set:

$$
\overline{\bar{\theta}}(x, t)=t{\overline{\theta\left(x, u_{1}\right)}}^{t} \quad \text { and } \quad \overline{\bar{\theta}}(t, x)={\overline{u_{2}}}^{t}{\overline{\theta\left(u_{1}, x\right)}}^{t} .
$$

So $\overline{\bar{\theta}}$ is well-defined and gives to $\overline{\bar{M}}$ a right-complemented presentation $\left\langle A \sqcup\{t\}: R_{\overline{\bar{\theta}}}\right\rangle^{+}$. 
The syntactic right-complement $\overline{\bar{\theta}}$ is defined on $(A \sqcup\{t\})^{2}$ and, by Lemma 2.21 , it can be uniquely extended by using

$$
\overline{\bar{\theta}}(u, v w)=\overline{\bar{\theta}}(u, v) \overline{\bar{\theta}}(\overline{\bar{\theta}}(v, u), w) \quad \text { and } \quad \overline{\bar{\theta}}(v w, u)=\overline{\bar{\theta}}(w, \overline{\bar{\theta}}(v, u))
$$

for any $u, v, w \in(A \sqcup\{t\})^{*}$. The diagrammatic mechanisms associated with a syntactic complement turn out to be essentially invariant under the stuffing operation. Indeed, we prove that

$$
\overline{\bar{\theta}}\left(\bar{u}^{t}, \bar{v}^{t}\right)=t \overline{\theta(u, v)}^{t} \quad(\overline{\bar{\theta}} \text {-invariance })
$$

holds for $u, v \in A^{+}$. We use induction on the number $n$ of steps of computation. For $n=1$, hence $|u|=|v|=1$, we have $\left(\bar{u}^{t}, \nabla^{t}\right)=(u, v) \in A^{2}$ and the result follows by definition.

Assume $n>1$ and, without loss of generality, $v=x w$ with $x \in A$ and $w \neq \varepsilon$, the $n$ steps decompose into $n_{1}+1+n_{2}$ steps according to the following diagram

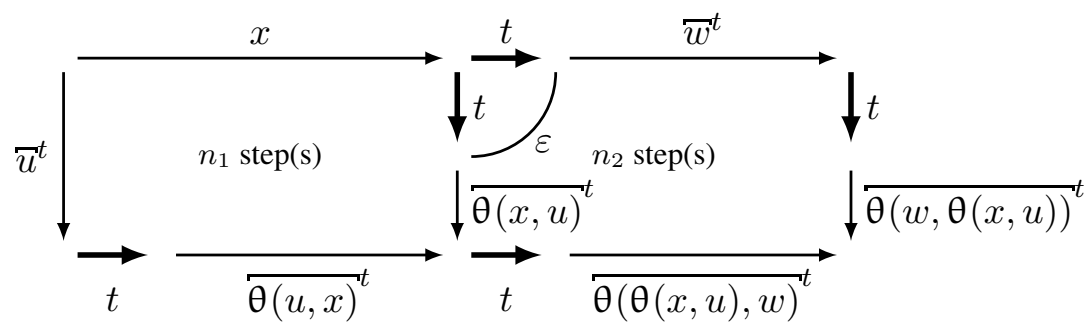

and we obtain

$$
\begin{aligned}
\overline{\bar{\theta}}\left(\bar{v}^{t}, \bar{u}^{t}\right) & =\overline{\bar{\theta}}\left(\overline{x w}^{t}, \bar{u}^{t}\right) & \overline{\bar{\theta}}\left(\bar{u}^{t}, \bar{v}^{t}\right) & =\overline{\bar{\theta}}\left(\bar{u}^{t}, \overline{x w}^{t}\right) \\
& =\bar{\theta}\left(x t \bar{w}^{t}, \bar{u}^{t}\right) & & \bar{\theta}\left(\bar{u}^{t}, x t \bar{w}^{t}\right) \\
& \stackrel{(\mathrm{IH})}{=} t \overline{\theta(w, \theta(x, u))}^{t} & & \stackrel{(\mathrm{IH})}{=} t \overline{\theta(u, x)} t \overline{\theta(\theta(x, u), w}^{t} \\
& =t \overline{\theta(x w, u)}^{t} & & =t \overline{\theta(u, x)}^{t}(\overline{\theta(x, u), w)} \\
& =t \overline{\theta(v, u)}^{t} & & =t \overline{\theta(u, v)}^{t},
\end{aligned}
$$

which concludes the induction. Similarly, we could find

$$
\overline{\bar{\theta}}\left(\bar{u}^{t}, \bar{v}^{t} t\right)=t \overline{\theta(u, v)}^{t} \quad \text { and } \quad \overline{\bar{\theta}}\left(\bar{v}^{t} t, \bar{u}^{t}\right)=\overline{\theta(v, u)}^{t} .
$$

The point is now to check the $\overline{\bar{\theta}}$-cube condition. Since $\overline{\bar{M}}$ is atomic, we only need to check it on $A \sqcup\{t\}$. It suffices to consider the two following cases, since the others are either symmetric or trivial.

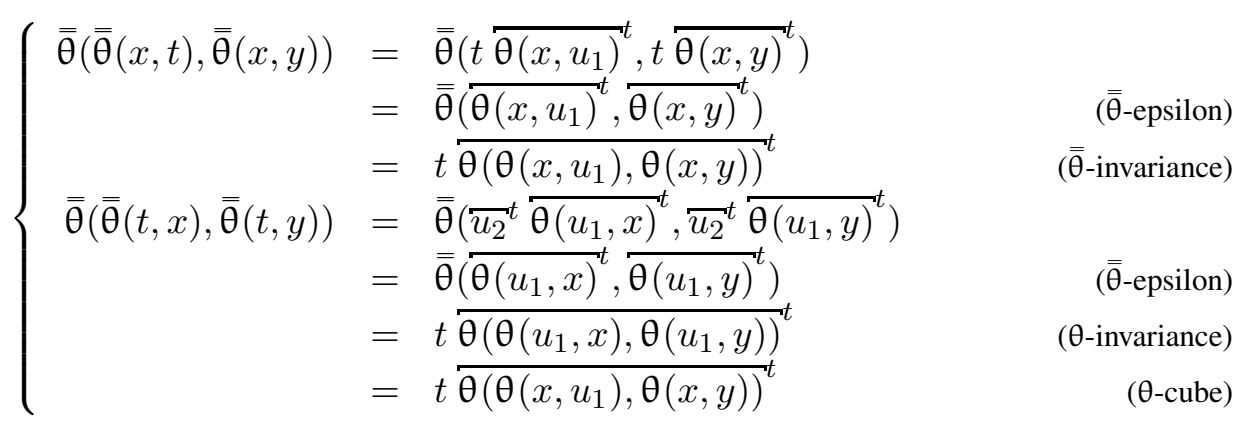




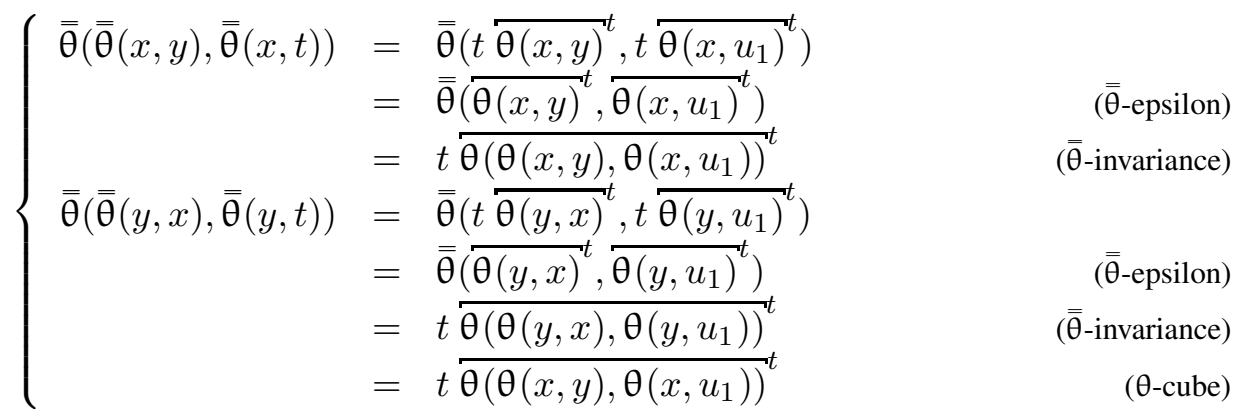

According to Theorem 2.24, $\overline{\bar{M}}$ is therefore a cancellative monoid admitting conditional lcms.

Here is the point where the hypothesis on the exponents enters the scene. The existence of a (central) Garside element is what is needed to conclude that $\bar{M}$ is a Garside monoid whose group of fractions is the enveloping group $\bar{G}$. Indeed, let $z$ denote some central Garside element of $M$. The hypothesis $\epsilon\left(h_{1}\right)=\epsilon\left(h_{2}\right)$ implies that the centre is nontrivial and then that $\psi(z)=z^{t} t$ is a central Garside element in $\overline{\bar{M}}$, concluding the proof.

Remark 4.3. The additional assumption $\left\|\phi_{1}(h)\right\|=\left\|\phi_{2}(h)\right\|$ can be naturally expressed more generally as the existence of any norm $v$ satisfying Lemma2.8(i).

Whenever the monoid $M$ admits an additive norm $v_{+}$, such an assumption becomes superfluous, thanks to the required condition on exponents: $h_{1}^{\ell}=h_{2}^{\ell}$ implies $v_{+}\left(h_{1}\right)=v_{+}\left(h_{2}\right)$.

On the contrary, Example 4.5 below illustrates various behaviors of cyclic $\mathrm{HNN}$ extensions of $M_{\mathrm{K}}$ possibly depending on this additional assumption.

Example 4.4. The simplest examples are those well-known Baumslag-Solitar groups

$$
\mathbf{B S}(m, m)=\left\langle\mathbf{s}, \mathbf{t}: \mathbf{s}^{m} \mathbf{t}=\mathrm{ts}^{m}\right\rangle,
$$

which are cyclic HNN extension of the infinite cyclic group. Known as Baumslag-Solitar monoids (see [8, 28, 43]), the associated monoids $\mathbf{B S}^{+}(m, m)=\left\langle\mathrm{s}, \mathrm{t}: \mathrm{s}^{m} \mathrm{t}=\mathrm{t} \mathbf{s}^{m}\right\rangle^{+}$happen to be cancellative atomic monoids admitting conditional lcms, but fail to be Garside monoids for $m>1$. Now, for any for $m>1$, the t-stuffed version (that is, the submonoid of $\psi\left(\mathbf{B S}^{+}(m, m)\right)$ generated by $\{\mathrm{s}, \mathrm{t}\})$ turns out to coincide with a dihedral Artin-Tits monoid

$$
\mathbf{B}^{+}\left(\mathbf{I}_{2}(2 m)\right)=\left\langle\mathbf{s}, \mathbf{t}:(\mathbf{s t})^{m}=(\mathrm{ts})^{m}\right\rangle^{+}
$$

which is Garside and embeds into its group of fractions $\mathbf{B S}(m, m)$ for $m>0$.

Example 4.5. Much more complicated examples are provided by the slightly pathological Garside monoid $M_{\mathrm{K}}=\langle\mathrm{x}, \mathrm{y}: \text { xyxyx }=\mathrm{yy}\rangle^{+}$from Example 2.10, Recall here that $M_{\mathrm{K}}$ admits no additive norm, that is, no norm $v$ satisfying $v(a b)=v(a)+v(b)$ for any $(a, b) \in M_{\mathrm{k}}$. Its minimal Garside element $\Delta=\mathrm{y}^{3}$ admits three cube roots while $\Delta^{2}$ admits seven:

$$
\Delta^{\frac{1}{3}}=\{y, x y, y x\} \quad \text { and } \Delta^{\frac{2}{3}}=\{x y x y, y x y x, y y, x x y x y, y x x y x, y x x y x, y x y x x\} .
$$

We have $\|\mathrm{y}\|=1,\|\mathrm{xy}\|=\|\mathrm{yx}\|=2,\|\operatorname{xyxy}\|=\|\mathrm{yxyx}\|=4,\|\mathrm{yy}\|=\|\operatorname{xxyxy}\|=\|\operatorname{yxxyx}\|=$ $\|\operatorname{yxxyx}\|=\|\operatorname{yxyxx}\|=5$, and $\left\|\mathrm{y}^{3}\right\|=6$.

Table 2 gathers the numbers of the possible cyclic HNN extensions of $M_{\mathrm{K}}$ when one arbitrarily restricts a pair $\left(h_{1}, h_{2}\right)$ of images of $h$ to be chosen from the set $\Delta^{\frac{p}{q}}$ with $\frac{p}{q}=\left\{\frac{1}{3}, \frac{1}{2}, \frac{1}{1}\right\}$. 

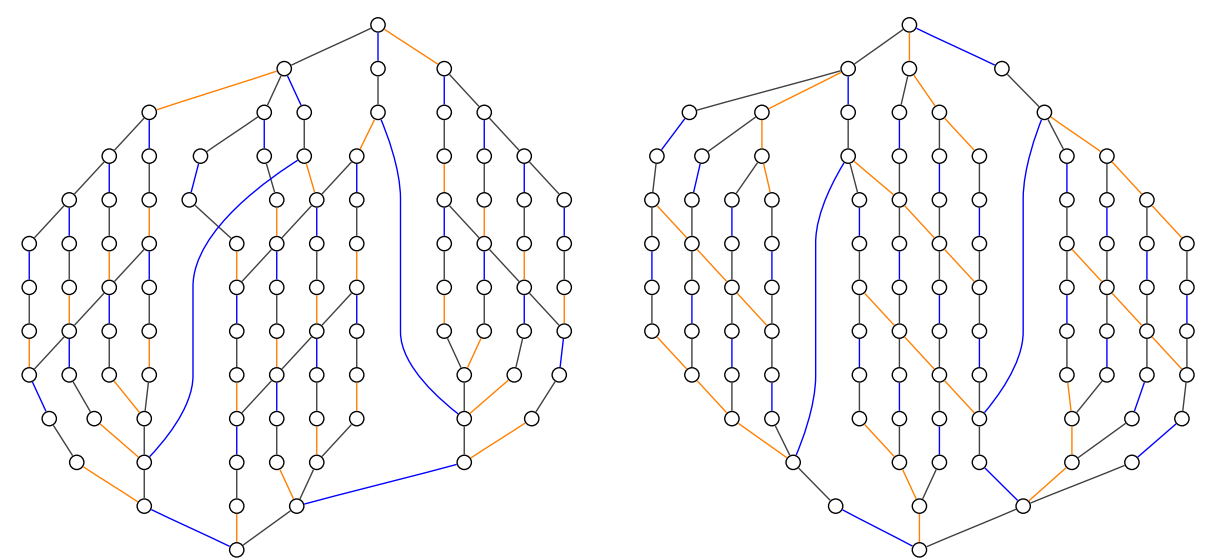

FIGURE 6. See Example 4.5, the (non-isomorphic) 96-simple lattices of $\bar{M}_{\mathrm{k}, \mathrm{xy}, \mathrm{xy}}=\langle\mathrm{x}, \mathrm{y}, \mathrm{z}: \mathrm{xzyzxzyzx}=\mathrm{yzy}, \mathrm{xzyz}=\mathrm{zxzy}\rangle^{+}$(anti-isomorphic to $\left.\overline{\bar{M}}_{\mathrm{k}, \mathrm{yx}, \mathrm{yx}}\right)$ and of $\overline{\bar{M}}_{\mathrm{k}, \mathrm{xy}, \mathrm{yx}}=\langle\mathrm{x}, \mathrm{y}, \mathrm{z}: \mathrm{xzyzxzyzx}=\mathrm{yzy}, \mathrm{xzyz}=\mathrm{zyzx}\rangle^{+}$ (anti-isomorphic to $\overline{\bar{M}}_{\mathrm{k}, \mathrm{yx}, \mathrm{xy}}$ ).

The nine cases corresponding with the restriction to $\Delta^{\frac{1}{3}}$ seem to catch at a glance the whole picture. For $\left(h_{1}, h_{2}\right)=(g, g)$ with $g \in \Delta^{\frac{1}{3}}$, the associated cyclic HNN extensions admit zstuffed presentations defining Garside monoids $\overline{\bar{M}}_{\mathrm{K}, g, g}$ (with respectively 88,96, and 96 simples). The latter two are non-isomorphic, but anti-isomorphic. The monoid $\bar{M}_{\mathrm{K}, \mathrm{yx}, \mathrm{xy}}$ is Garside (with 96 simples), is neither isomorphic nor anti-isomorphic to any of the latter two, now it is antiisomorphic to the monoid $\overline{\bar{M}}_{\mathrm{k}, \mathrm{xy}, \mathrm{yx}}$ (see Figure 6 ). The monoid $\overline{\bar{M}}_{\mathrm{k}, \mathrm{xy}, \mathrm{y}}$ is clearly non-atomic, now its enveloping group also envelops the Garside monoid $\bar{M}_{\mathrm{k}, \mathrm{y}, \mathrm{xy}}$. Figure 7 displays its 126simple lattice, whose anti-isomorphic image corresponds to the (Garside) monoid $\overline{\bar{M}}_{\mathrm{K}, \mathrm{yx}, \mathrm{y}}$ (whose enveloping group of fractions also envelops the non-atomic monoid $\overline{\bar{M}}_{\mathrm{K}, \mathrm{y}, \mathrm{yx}}$ ).

The previous result can be fully applied to the class of tree products of infinite cyclic groups. Gathering Corollary 3.10 and Theorem 4.2 , we obtain:

Corollary 4.6. Let $\mathcal{T}$ be a finite weighted tree. For any vertices $x, z \in V(\mathcal{T})$, the cyclic $H N N$ extension $\langle G(\mathcal{T}), t: t x=z t\rangle$ is Garside if and only if the weighted path $x \frac{p_{1} q_{1}}{\cdots} \cdots \frac{p_{m} q_{m}}{z}$ satisfies $p_{1} \cdots p_{m}=q_{1} \cdots q_{m}$.

Let us first state a straightforward fact about exponents.

Lemma 4.7. Let $\mathcal{T}$ be a finite weighted tree with weights in $\{2,3,4 \ldots\}$. For any adjacent vertices $x, z \in V(\mathcal{T})$ with $x \frac{p q}{z}$, the exponents of $x$ and $z$ satisfy $\frac{\epsilon(x)}{p}=\frac{\epsilon(z)}{q}$.

Proof. Recall first that, under the mild hypothesis that the weights all belong to $\{2,3,4 \ldots\}$, the monoid $G^{+}(\mathcal{T})$ is Garside by (the proof of) Corollary 3.10. Then, by definition of $\epsilon$ and by hypothesis, we have

$$
\left(x^{\epsilon(x)}\right)^{q} \stackrel{\text { def }}{=}\left(z^{\epsilon(z)}\right)^{q}=\left(z^{q}\right)^{\epsilon(z)} \stackrel{\text { hyp }}{=}\left(x^{p}\right)^{\epsilon(z)} .
$$

We conclude $\frac{\epsilon(x)}{p}=\frac{\epsilon(z)}{q}$ by invoking the torsion-freeness of any Garside monoid. 


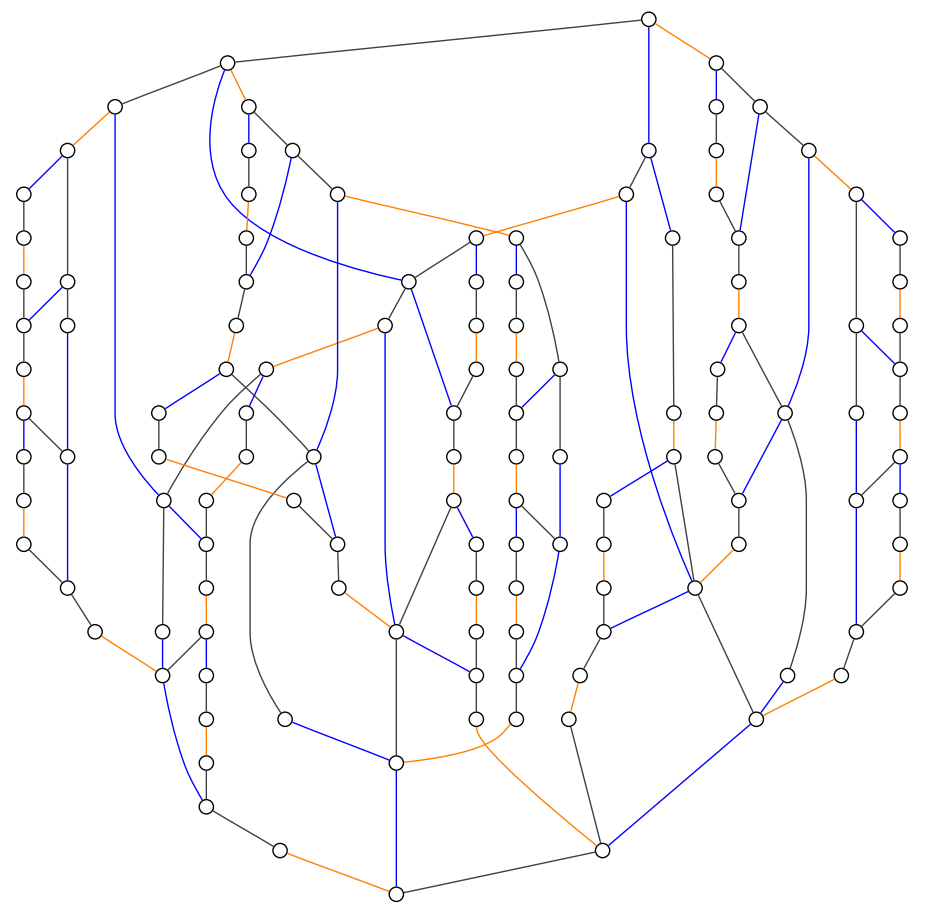

Figure 7. See Example 4.5, the 126-simple lattices of $\bar{M}_{\mathrm{k}, \mathrm{y}, \mathrm{xy}}=\langle\mathrm{x}, \mathrm{y}, \mathrm{z}$ : xzyzxzyzx $=\mathrm{yzy}, \mathrm{yz}=\mathrm{zxzy}\rangle^{+}\left(\right.$anti-isomorphic to $\left.\overline{\bar{M}}_{\mathrm{k}, \mathrm{yx}, \mathrm{y}}\right)$.

\begin{tabular}{|c|c|c|c|c|c|c|c|c|c|c|c|c|c|}
\hline & & & \multicolumn{3}{|c|}{$\Delta^{\frac{1}{3}}$} & \multicolumn{7}{|c|}{$\Delta^{\frac{2}{3}}$} & $\Delta^{\frac{1}{1}}$ \\
\hline & & $h_{2}$ & $\mathrm{y}$ & xy & $\mathrm{yx}$ & xyxy & yxyx & yy & xxyxy & xyxxy & yxxyx & yxyxx & yyy \\
\hline & $h_{1}$ & $\left\|h_{i}\right\|$ & 1 & 2 & 2 & 4 & 4 & 5 & 5 & 5 & 5 & 5 & 6 \\
\hline \multirow{3}{*}{$\Delta^{\frac{1}{3}}$} & $\mathrm{y}$ & 1 & 88 & 126 & $\infty$ & & & & & & & & \\
\hline & $x y$ & 2 & $\infty$ & 96 & 96 & & & & & & & & \\
\hline & $\mathrm{yx}$ & 2 & 126 & 96 & 96 & & & & & & & & \\
\hline \multirow{7}{*}{$\Delta^{\frac{2}{3}}$} & xyxy & 4 & & & & 2304 & 2304 & $\infty$ & 1561 & 1687 & 1561 & 1687 & \\
\hline & yxyx & 4 & & & & 2304 & 2304 & 2552 & 1687 & 1561 & 1561 & $\infty$ & \\
\hline & yy & 5 & & & & 2552 & $\infty$ & 1808 & 1109 & 1109 & 1109 & 1109 & \\
\hline & xxyxy & 5 & & & & $\infty$ & 1687 & 1109 & 804 & 804 & 804 & 804 & \\
\hline & xyxxy & 5 & & & & 1561 & 1561 & 1109 & 804 & 804 & 804 & 804 & \\
\hline & yxxyx & 5 & & & & 1561 & 1687 & 1109 & 804 & 804 & 804 & 804 & \\
\hline & yxyxx & 5 & & & & 1687 & 1561 & 1109 & 804 & 804 & 804 & 804 & \\
\hline$\Delta^{\frac{1}{1}}$ & yyy & 6 & & & & & & & & & & & 44 \\
\hline
\end{tabular}

TABLE 2. See Example 4.5, the number of simples of the Garside monoid $\overline{\bar{M}}_{\mathrm{K}}$ for these HNN extensions of $M_{\mathrm{K}}$ with $h_{i}=\phi_{i}(h) \in \Delta^{\frac{1}{3}} \sqcup \Delta^{\frac{2}{3}} \sqcup \Delta^{\frac{1}{1}}$ for $i \in\{1,2\}$. Gray cells correspond to pairs $\left(h_{1}, h_{2}\right)$ with $\left\|h_{1}\right\| \neq\left\|h_{2}\right\|$, while the symbol $\infty$ indicates when $\bar{M}_{\mathrm{K}}$ is effectively non-atomic. 
There are many equivalent ways to compute the exponents of the vertices. We can start from the initial positively reduced tree $\mathcal{T}$ and complete it into the associated weighted complete graph $\mathrm{K}_{\mathcal{T}}$ by successive triangulations: whenever $x \frac{a b}{b} y$ and $y \frac{c d}{z} z$ are edges, we add the edge

$$
x \frac{a \frac{c \vee b}{b} \quad d \frac{b \vee c}{c}}{z}
$$

if needed, according to Lemma 4.7. The exponent $\epsilon(v)$ of any vertex $v$ is then obtained by taking the lcm of all these weights $p$ with $v \frac{p \quad q}{v^{\prime}}$ an edge in $\mathrm{K}_{\mathcal{T}}$.

Instead of completing $\mathcal{T}$ into $\mathrm{K}_{\mathcal{T}}$, we can also choose to compute the exponent of any vertex $v$ by rooting the tree $\mathcal{T}_{v}$ at $v$ and by exploring the obtained rooted tree $\mathcal{T}_{v}$. We have:

Lemma 4.8. Let $\mathcal{T}$ be a finite weighted tree with weights in $\{2,3,4 \ldots\}$. The exponent $\epsilon(v)$ of a vertex $v \in V(\mathcal{T})$ can be obtained as $\lambda\left(\mathcal{T}_{v}\right)$ where $\lambda$ is recursively defined by $\lambda(x)=1$ for $x$ a leaf and by

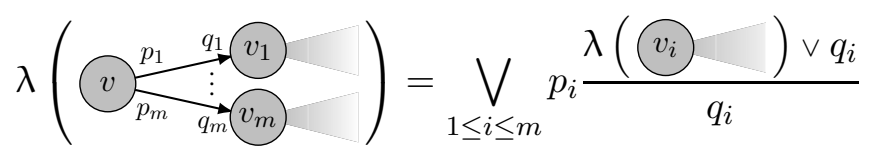

otherwise.

Proof. The point is that, for any vertex $x \in V(\mathcal{T})$, the value of $\lambda(x)$ coincides with the exponent of the vertex $x$ in the submonoid generated by the subtree pending from this root $x$. Up to duplicating some vertices, we can suppose that $\mathcal{T}_{v}$ is either a linear or a starlike tree with root $v$. We use an induction on the number $m \geq 1$ of branches of this starlike tree. For $m=1$, we use an induction on the length $h \geq 1$ of the linear rooted tree For $h=1$, we find $\epsilon(v)=\lambda\left(v \stackrel{p_{1}}{\left.\stackrel{q_{1}}{\longrightarrow} v_{1}\right)}\right)=p_{1} \frac{\lambda\left(v_{1}\right) \vee q_{1}}{q_{1}}=p_{1} \frac{1 \vee q_{1}}{q_{1}}=p_{1}$ as expected. Assume $h>1$, the exponent of the vertex $v_{1}$ in the submonoid generated by the subtree pending from $v_{1}$ is $\left.\lambda\left(v_{1}\right]\right)$ by induction hypothesis. We obtain

$$
\epsilon(v)=\lambda(\vartheta)=p_{1} \frac{\lambda\left(v_{1}\right) \vee q_{1}}{q_{1}},
$$

which concludes the induction on $h \geq 1$.

Assume $m>1$ and let $f_{i}=p_{i} \frac{\lambda\left(v_{i}\right) \vee q_{i}}{q_{i}}$ for $1 \leq i \leq m$. The exponent of $v$ in $G^{+}(\mathcal{T})$ then coincides with the exponent of $v^{\prime}$ in the monoid $G^{+}\left(\mathcal{T}^{\prime}\right)$ generated by the star $\mathcal{T}^{\prime}$ below:

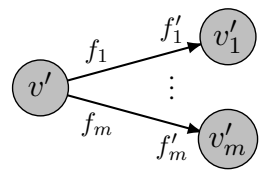

which concludes the induction on $m \geq 1$ and the proof.

Proof of Corollary 4.6 By Corollary 3.10, $G(\mathcal{T})$ is indeed a Garside group. According to Theorem 4.2, it remains to verify that the two vertices $x, z$ satisfy $\epsilon(x)=\epsilon(z)$ if and only if the 
weighted path $x=v_{0} \stackrel{p_{1}}{q_{1}} v_{1} \cdots v_{m-1} \frac{p_{m} \quad q_{m}}{v_{m}}=z$ satisfies $p_{1} \cdots p_{m}=q_{1} \cdots q_{m}$. Note that the latter equality is invariant under both atomic and positive transformations, that we defined for the proof of Corollary 3.10 . So we can assume from now on that the weights of the considered tree $\mathcal{T}$ all belong to $\{2,3, \ldots\}$.

Rooting $\mathcal{T}$ at $x=v_{0}$, we consider the residual subtrees $\mathcal{R}_{v_{i}}$ rooted at each vertex $v_{i}$ for $0 \leq i \leq m$ according to the following combing

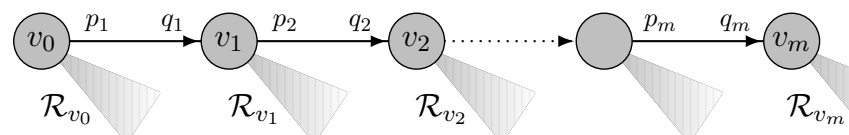

that is, the subtrees $\mathcal{R}_{v_{i}}$ that we would obtain after erasing from $\mathcal{T}$ each edge between $v_{i-1}$ and $v_{i}$ for $1 \leq i \leq m$. Note that the definition of these rooted subtrees $\mathcal{R}_{v_{i}}$ does not depend on the choice of the original root for $\mathcal{T}$, provided that such a root is chosen among the vertices $v_{i}$ along the path between $x$ and $z$. We shall compare $\epsilon\left(v_{0}\right)$ and $\epsilon\left(v_{m}\right)$ by using the auxiliary map $\lambda$ and Lemma4.8.

Let $r_{i}$ denote $\lambda\left(\begin{array}{c}v_{i} \\ \mathcal{R}_{v_{i}}\end{array}\right)$ for $0 \leq i \leq m$ and $e_{i} \operatorname{denote} \lambda\left(\begin{array}{c}v_{i} \stackrel{p_{i}}{\longrightarrow} \\ \mathcal{R}_{v_{i}}\end{array}\right)$ for $0 \leq i<m$. We now show $e_{0}=r_{0} \vee \frac{1}{q_{1} \cdots q_{m}} \bigvee_{1 \leq i \leq m} p_{1} \cdots p_{i-1} p_{i}\left(q_{i} \vee r_{i}\right) q_{i+1} \cdots q_{m}$ by induction on $m \geq 0$. The result is obvious for $m=0$. Assume $m>0$. We find first $\epsilon\left(v_{0}\right)=e_{0}=r_{0} \vee p_{1} \frac{q_{1} \vee e_{1}}{q_{1}}$ by Lemma 4.8 and then

$$
\begin{aligned}
e_{0} & \stackrel{(\mathrm{IH})}{=} r_{0} \vee p_{1} \frac{q_{1} \vee\left(r_{1} \vee \frac{1}{q_{2} \cdots q_{m}} \vee_{2 \leq i \leq m} p_{2} \cdots p_{i-1} p_{i}\left(q_{i} \vee r_{i}\right) q_{i+1} \cdots q_{m}\right)}{q_{1}} \\
& =r_{0} \vee \frac{p_{1}}{q_{1} q_{2} \cdots q_{m}}\left(\left(q_{1} \vee r_{1}\right) q_{2} \cdots q_{m} \vee \bigvee_{2 \leq i \leq m} p_{2} \cdots p_{i-1} p_{i}\left(q_{i} \vee r_{i}\right) q_{i+1} \cdots q_{m}\right) \\
& =r_{0} \vee \frac{1}{q_{1} q_{2} \cdots q_{m}}\left(p_{1}\left(q_{1} \vee r_{1}\right) q_{2} \cdots q_{m} \vee \bigvee_{2 \leq i \leq m} p_{1} p_{2} \cdots p_{i-1} p_{i}\left(q_{i} \vee r_{i}\right) q_{i+1} \cdots q_{m}\right) \\
& =r_{0} \vee \frac{1}{q_{1} q_{2} \cdots q_{m}} \bigvee_{1 \leq i \leq m} p_{1} \cdots p_{i-1} p_{i}\left(q_{i} \vee r_{i}\right) q_{i+1} \cdots q_{m},
\end{aligned}
$$

which concludes the induction. By just introducing a dummy weight $q_{0}=1$, we obtain the completely homogeneous formula

$$
\epsilon(x)=\epsilon\left(v_{0}\right)=\frac{1}{q_{1} q_{2} \cdots q_{m}} \bigvee_{0 \leq i \leq m} p_{1} \cdots p_{i}\left(q_{i} \vee r_{i}\right) q_{i+1} \cdots q_{m}
$$

We obtain symmetrically

$$
\epsilon(z)=\epsilon\left(v_{m}\right)=\frac{1}{p_{1} p_{2} \cdots p_{m}} \bigvee_{0 \leq i \leq m} q_{m} \cdots q_{i+1}\left(p_{i+1} \vee r_{i}\right) p_{i} \cdots p_{1},
$$


where $p_{m+1}=1$ is also used as some dummy weight. By using the symmetry of the just obtained iterative versions, we deduce

$$
\epsilon\left(v_{0}\right)=\epsilon\left(v_{m}\right) \Longleftrightarrow p_{1} \cdots p_{m}=q_{1} \cdots q_{m},
$$

which concludes the proof.

Example 4.9. In Figure 5, each vertex $v \in V\left(\mathcal{T}_{0}^{\prime}\right)$ has been labelled with the value $\epsilon(v)$. So, in addition to the seven direct HNN extensions corresponding to loops (that is, with $x=z$ ), Theorem 4.2 and Corollary 4.6 allow to foresee that exactly two single cyclic HNN extensions are Garside (namely, $\epsilon(x)=\epsilon(z) \in\{48,60\}$ ). Multiple cyclic HNN extensions (for instance, $\epsilon(x)=\epsilon(z)=48$ plus $\left.\epsilon\left(x^{\prime}\right)=\epsilon\left(z^{\prime}\right)=60\right)$ provide again Garside groups.

All the arguments advanced here can be carefully revisited in order to obtain an even more general result that a Generalised Baumslag-Solitar group is Garside if and only if its centre is nontrivial, which essentially seems that a GBS group is Garside whenever it is a GBS-tree group [30]. We refer to [19] for a recent, independent and global approach of the calculus of the centre of GBS groups (see also [46]).

Finally, the possible mechanism-if $\overline{\bar{M}}_{\mathrm{\kappa}, h_{1}, h_{2}}$ is not atomic, then $\overline{\bar{M}}_{\mathrm{\kappa}, h_{2}, h_{1}}$ is atomic-observed for instance in Example 4.5 and Table 2, could lead to raise the question of weakening or even skipping the length assumption in Theorem 4.2. However, as already mentioned in Example 4.4, the Baumslag-Solitar group $\mathbf{B S}(m, n)=\left\langle\mathbf{s}, \mathbf{t}: \mathbf{s}^{m} \mathbf{t}=\mathbf{t} \mathbf{s}^{n}\right\rangle$ is a Garside group if and only if $\mathbf{B S}(m, n)$ is an automatic group if and only if $m=n$ holds (see [21, Example 7.4.1]).

\section{PIETROWSKI GROUPS}

Using the solution of Pietrowski for the isomorphism problem for one-relator groups with nontrivial centre [44], we finally state that a non-cyclic one-relator group is Garside if and only if its centre is nontrivial.

Theorem 5.1. [44, Theorems 1 and 3] Assume that $G$ is a non-cyclic one-relator group with nontrivial centre. Then, if $G /[G, G]$ is not free abelian, $G$ can be uniquely presented as

$$
\left\langle\mathrm{a}_{1}, \mathrm{a}_{2}, \ldots, \mathrm{a}_{m}: \mathrm{a}_{1}^{p_{1}}=\mathrm{a}_{2}^{q_{1}}, \ldots, \mathrm{a}_{m-1}^{p_{m-1}}=\mathrm{a}_{m}^{q_{m-1}}\right\rangle
$$

with $p_{i}, q_{i} \geq 2$ and $p_{i} \wedge q_{j}=1$ for $i>j$, and, if $G /[G, G]$ is free abelian, $G$ can be uniquely 2 presented as

$$
\left\langle\mathrm{a}, \mathrm{a}_{1}, \mathrm{a}_{2}, \ldots, \mathrm{a}_{m}: \mathrm{aa}_{1}=\mathrm{a}_{m} \mathrm{a}, \mathrm{a}_{1}^{p_{1}}=\mathrm{a}_{2}^{q_{1}}, \ldots, \mathrm{a}_{m-1}^{p_{m-1}}=\mathrm{a}_{m}^{q_{m-1}}\right\rangle
$$

with $p_{i}, q_{i} \geq 2, p_{i} \wedge q_{j}=1$ for $i>j$, and $p_{1} p_{2} \cdots p_{m-1}=q_{1} q_{2} \cdots q_{m-1}$.

Definition 5.2. A group is called an m-Pietrowski group if it admits (exactly) one of the presentations of Theorem 5.1 for some (unique) integer $m>1$.

The just-above term can be viewed as a short for what we could call a Baumslag-Collins-KarrassMcCool-Meskin-Metaftsis-Magnus-Murasugi-Pietrowski-Solitar-Steinberg-Taylor group.

\footnotetext{
1 modulo mirror symmetry

2 modulo exchange and cyclic permutations
} 
We gather three properties (see [10,33, 34]) illustrating the still mysterious distribution of onerelator groups with nontrivial centre within the class of Pietrowski groups.

- Every $m$-Pietrowski group with $m \leq 3$ is a one-relator group: the group

$$
\left\langle\mathrm{a}_{1}, \mathrm{a}_{2}, \mathrm{a}_{3}: \mathrm{a}_{1}^{p_{1}}=\mathrm{a}_{2}^{q_{1}}, \mathrm{a}_{2}^{p_{2}}=\mathrm{a}_{3}^{q_{2}}\right\rangle
$$

with $p_{i}, q_{i} \geq 2$ and $q_{1} \wedge p_{2}=1$ is isomorphic to the group

$$
\left\langle\mathrm{a}_{1}, \mathrm{a}_{3}: \pi_{p_{2}, q_{1}}\left(\mathrm{a}_{1}^{p_{1}}, \mathrm{a}_{3}^{-q_{2}}\right)\right\rangle,
$$

where $\pi_{\lambda, \mu}(x, y)$ denotes the unique primitive element (up to conjugation) with exponent sum $\lambda$ on $x$ and $\mu$ on $y$ (note that there is a misprint in the original statement of [34]).

- Any group $\left\langle a_{1}, a_{2}, a_{3}, a_{4}: a_{1}^{2}=a_{2}^{2}, a_{2}^{p_{2}}=a_{3}^{p_{2}}, a_{3}^{3}=a_{4}^{3}\right\rangle$ with $p_{2} \geq 2$ happens to not be a one-relator group.

- For every $m$, there exists an $m$-Pietrowski group which is a one-relator group.

Applying Corollaries 3.10 and 4.6 , we deduce these rather amazing facts:

Corollary 5.3. Every Pietrowski group is Garside.

Corollary 5.4. A non-cyclic one-relator group is Garside if and only if its centre is nontrivial.

Note that Baumslag and Taylor [2] have given an algorithm for deciding whether or not a onerelator group has a nontrivial centre. By Corollary [5.4, one can decide whether or not a given one-relator group is a Garside group.

Example 5.5. Figure 8 displays the 1353-simple lattice of the Garside structure for the one-relator group

$$
\left\langle a, x: x^{8} a x^{-6} a^{-1} x^{4} a x^{-6} a^{-1}\right\rangle,
$$

whose associated Pietrowski presentation is

$$
\left\langle\mathrm{a}, \mathrm{x}_{1}, \mathrm{x}_{2}, \mathrm{x}_{3}: \mathrm{ax}_{1}=\mathrm{x}_{3} \mathrm{a}, \mathrm{x}_{1}^{4}=\mathrm{x}_{2}^{2}, \mathrm{x}_{2}^{3}=\mathrm{x}_{3}^{6}\right\rangle .
$$

Interestingly, such a Garside structure provides an explicit biautomatic structure [12, 40, 42].

Corollary 5.6. All Pietrowski groups and, in particular, all (non-cyclic) one-relator groups with nontrivial centre are biautomatic and have solvable conjugacy problem.

All Pietrowski groups and, in particular, all (non-cyclic) one-relator groups with nontrivial centre are torsion-free. In particular, gathering Corollary 5.6 and the solution of Newman for one-relator groups with torsion [35], the conjugacy problem remains open for torsion-free one-relator groups with trivial centre (see [1, Problem (O5)] and also [31]).

\section{REFERENCES}

[1] Gilbert Baumslag, Alexei G. Myasnikov, and Vladimir Shpilrain. Open problems in combinatorial group theory. Second edition. In Combinatorial and geometric group theory, volume 296 of Contemp. Math., pages 1-38. Amer. Math. Soc., 2002.

[2] Gilbert Baumslag and Tekla Taylor. The centre of groups with one defining relator. Math. Ann., 175:315-319, 1968.

[3] David Bessis. The dual braid monoid. Ann. Sci. École Norm. Sup., 36(5):647-683, 2003.

[4] David Bessis, François Digne, and Jean Michel. Springer theory in braid groups and the Birman-Ko-Lee monoid. Pacific J. Math., 205(2):287-309, 2002. 


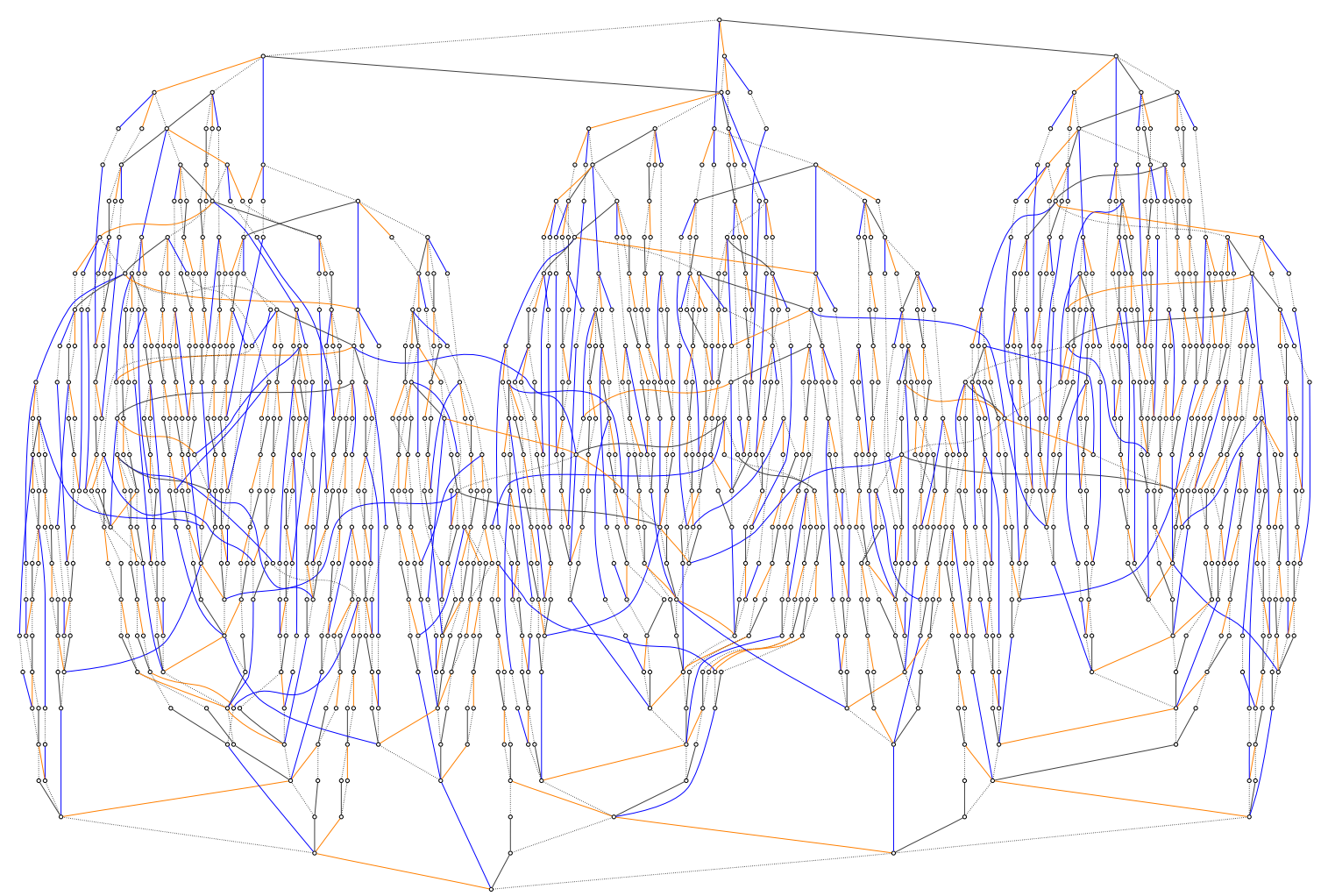

FIGURE 8. The 1353-simple lattice of the Garside structure for the one-relator group $\left\langle\mathrm{a}, \mathrm{x}: \mathrm{x}^{8} \mathrm{ax}^{-6} \mathrm{a}^{-1} \mathrm{x}^{4} \mathrm{ax}^{-6} \mathrm{a}^{-1}\right\rangle$ from Example 5.5.

[5] Joan Birman, Ki Hyoung Ko, and Sang Jin Lee. A new approach to the word and conjugacy problems in the braid groups. Adv. Math., 139(2):322-353, 1998.

[6] Egbert Brieskorn and Kyoji Saito. Artin-Gruppen und Coxeter-Gruppen. Invent. Math., 17:245-271, 1972.

[7] Michel Broué, Gunter Malle, and Raphaël Rouquier. Complex reflection groups, braid groups, Hecke algebras. J. Reine Angew. Math., 500:127-190, 1998.

[8] Alan J. Cain. Automatic structures for subsemigroups of Baumslag-Solitar semigroups. Semigroup Forum, 87(3):537-552, 2013.

[9] Alfred H. Clifford and Gordon B. Preston. The algebraic theory of semigroups. Vol. I. Mathematical Surveys, No. 7. Amer. Math. Soc., 1961.

[10] Donald J. Collins. Generation and presentation of one-relator groups with centre. Math. Z., 157(1):63-77, 1977.

[11] Ruth Corran and Matthieu Picantin. A new Garside structure for the braid groups of type (e,e,r). J. Lond. Math. Soc., 84(3):689-711, 2011.

[12] Patrick Dehornoy. Groupes de Garside. Ann. Sci. École Norm. Sup. (4), 35(2):267-306, 2002.

[13] Patrick Dehornoy. Complete positive group presentations. J. Algebra, 268(1):156-197, 2003.

[14] Patrick Dehornoy. The subword reversing method. Internat. J. Algebra Comput., 21(1-2):71-118, 2011.

[15] Patrick Dehornoy et al. Foundations of Garside theory, volume 22. Europ. Math. Soc. Tracts in Mathematics, 2015. URL: https://dehornoy.users.lmno.cnrs.fr/Books/Garside/Text.pdf

[16] Patrick Dehornoy and Luis Paris. Gaussian groups and Garside groups, two generalisations of Artin groups. Proc. London Math. Soc. (3), 79(3):569-604, 1999.

[17] Deko V. Dekov. The embedding of semigroup amalgams. J. Algebra, 141(1):158-161, 1991.

[18] Deko V. Dekov. Free products with amalgamation of monoids. J. Pure Appl. Algebra, 125(1-3):129-133, 1998. 
[19] Alberto L. Delgado, Derek J. S. Robinson, and Mathew Timm. Cyclic normal subgroups of generalized BaumslagSolitar groups. Communications in Algebra, 45(4):1808-1818, 2017.

[20] Pierre Deligne. Les immeubles des groupes de tresses généralisés. Invent. Math., 17:273-302, 1972.

[21] David B. A. Epstein, James W. Cannon, Derek F. Holt, Silvio V. F. Levy, Michael S. Paterson, and William P. Thurston. Word processing in groups. Jones and Bartlett Publishers, 1992.

[22] Frank A. Garside. The braid group and other groups. Quart. J. Math. Oxford Ser. (2), 20:235-254, 1969.

[23] Eddy Godelle and Luis Paris. PreGarside monoids and groups, parabolicity, amalgamation, and FC property. Internat. J. Algebra Comput., 23(6):1431-1467, 2013.

[24] Graham Higman, Bernhard H. Neumann, and Hanna Neumann. Embedding theorems for groups. J. London Math. Soc., 24:247-254, 1949.

[25] John M. Howie. Embedding theorems with amalgamation for semigroups. Proc. London Math. Soc. (3), 12:511534, 1962.

[26] John M. Howie. An embedding theorem with amalgamation for cancellative semigroups. Proc. Glasgow Math. Assoc., 6:19-26, 1963.

[27] John M. Howie. Embedding theorems for semigroups. Quart. J. Math. Oxford Ser. (2), 14:254-258, 1963.

[28] David A. Jackson. Decision and separability problems for Baumslag-Solitar semigroups. Internat. J. Algebra Comput., 12(1-2):33-49, 2002.

[29] Sang Jin Lee. Garside groups are strongly translation discrete. J. Algebra, 309(2):594-609, 2007.

[30] Gilbert Levitt. On the automorphism group of generalized Baumslag-Solitar groups. Geom. Topol., 11:473-515, 2007.

[31] Roger C. Lyndon and Paul E. Schupp. Combinatorial group theory. Springer-Verlag, Berlin-New York, 1977.

[32] Wilhelm Magnus, Abraham Karrass, and Donald Solitar. Combinatorial group theory. Dover Publications, Inc., New York, revised edition, 1976. Presentations of groups in terms of generators and relations.

[33] James McCool. A class of one-relator groups with centre. Bull. Aust. Math. Soc., 44(2):245-252, 1991.

[34] Stephen Meskin, Alfred Pietrowski, and Arthur Steinberg. One-relator groups with center. J. Austral. Math. Soc., 16:319-323, 1973. Collection of articles dedicated to the memory of Hanna Neumann.

[35] B. B. Newman. Some results on one-relator groups. Bull. Amer. Math. Soc., 74:568-571, 1968.

[36] Matthieu Picantin. Petits groupes gaussiens. PhD thesis, Univ Caen Normandie, 2000.

[37] Matthieu Picantin. The center of thin Gaussian groups. J. Algebra, 245(1):92-122, 2001.

[38] Matthieu Picantin. The conjugacy problem in small Gaussian groups. Comm. Algebra, 29(3):1021-1039, 2001.

[39] Matthieu Picantin. Explicit presentations for the dual braid monoids. C. R. Acad. Sci., 334(10):843-848, 2002.

[40] Matthieu Picantin. Automatic structures for torus link groups. J. Knot Theory Ramifications, 12(6):833-866, 2003.

[41] Matthieu Picantin. Finite transducers for divisibility monoids. Theoret. Comput. Sci., 362(1-3):207-221, 2006.

[42] Matthieu Picantin. Automates, (semi)groupes, dualités. Habilitation à diriger des recherches, Univ Paris Diderot, 2017. URL: http://www.irif.fr/ picantin/papers/hdr_memoire.pdf.

[43] Matthieu Picantin. Automatic semigroups vs automaton semigroups. In 46th International Colloquium on Automata, Languages, and Programming (ICALP 2019), volume 119 of LIPICs, pages 1-15, 2019.

[44] Alfred Pietrowski. The isomorphism problem for one-relator groups with non-trivial centre. Math. Z., 136:95-106, 1974.

[45] Jean-Philippe Préaux. Le problème de conjugaison dans le groupe d'une 3-variété orientable vérifiant la conjecture de géométrisation de Thurston. $\mathrm{PhD}$ thesis, Univ Marseille, 2001.

[46] Derek J. S. Robinson. Generalized Baumslag-Solitar groups: a survey of recent progress. In Groups St. Andrews 2013, volume 423 of London Math. Soc Lecture Notes, pages 457-468, 2015.

[47] Jean-Pierre Serre. Arbres, amalgames, $\mathrm{SL}_{2}$. Astérisque 46. SMF, Paris, 1977.

[48] Hervé Sibert. Extraction of roots in Garside groups. Comm. Algebra, 30(6):2915-2927, 2002.

[49] Vladimir B. Styšnev. Taking the root in the braid group. Izv. Akad. Nauk SSSR, 42(5):1120-1131, 1183, 1978.

[50] Jenö Szép. On the structure of groups which can be represented as the product of two subgroups. Acta Sci. Math. Szeged, 12:57-61, 1950.

[51] Guido Zappa. Sulla costruzione dei gruppi prodotto di due dati sottogruppi permutabili tra loro. In Atti Secondo Congresso Un. Mat. Ital., Bologna, 1940, pages 119-125. Edizioni Cremonense, 1942.

IRIF, UMR 8243 CNRS \& UNIV. PARIS DIDEROT, 75013 PARIS, FRANCE.

E-mail address: picantin@irif.fr 